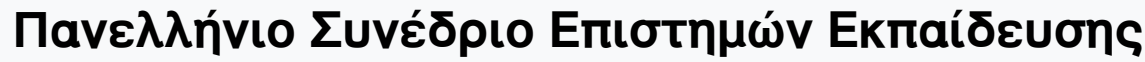

Tóp. 8 (2018)

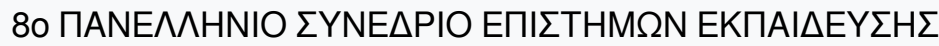

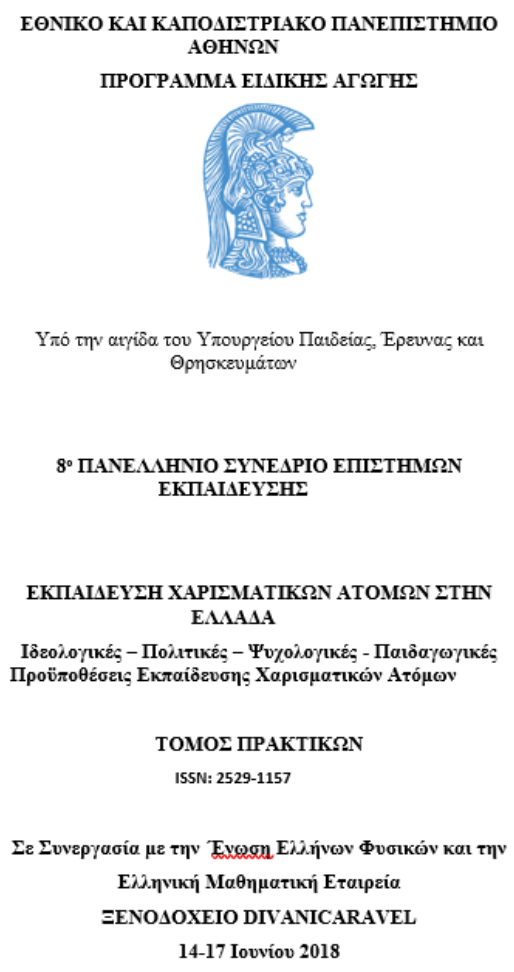

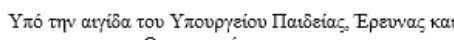

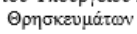

8 ПANEAAHNIO IYNEAPIO EIIITHMQN EKMAIAEYEHЕ

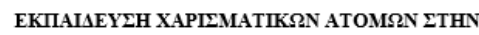
EAAA $\triangle A$

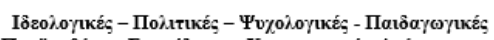

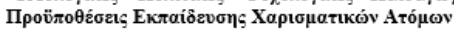

TOMOE IIPAKTIKSN

ISSN: 2529-1157

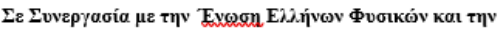

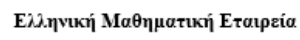

כENOAOXEIO DIVANICARAVEL

14-17 Iovvíov 2018

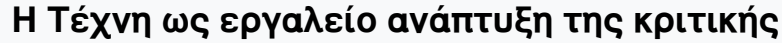

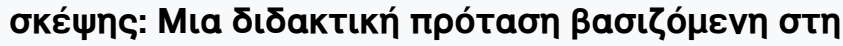

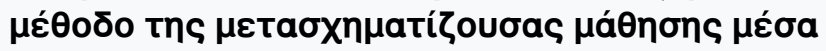

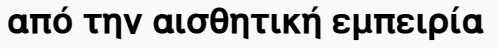

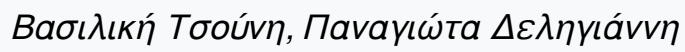

doi: $\underline{10.12681 / e d u s c .2807}$

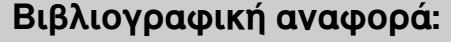

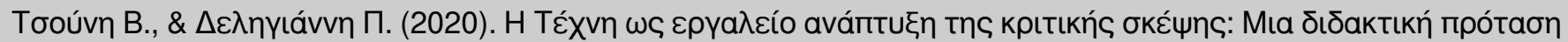

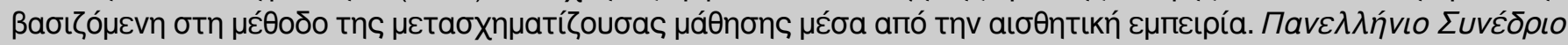

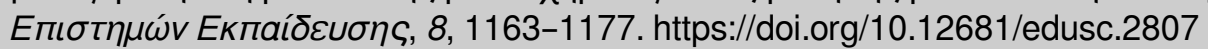




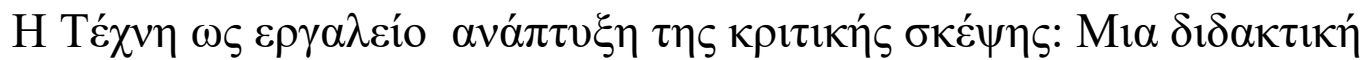

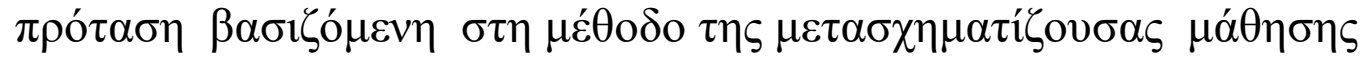

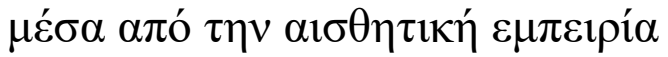

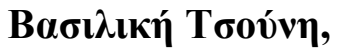

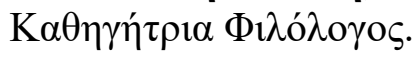 \\ vtsouni@yahoo.gr \\ $\Delta \varepsilon \lambda \eta \gamma \mathbf{\alpha} \mathbf{v v \eta} \Pi \alpha v \alpha \gamma i \omega ́ \tau \alpha$

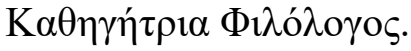

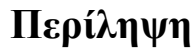

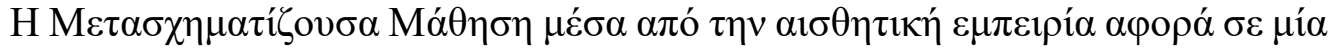

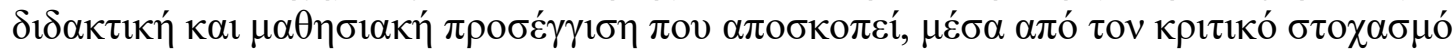

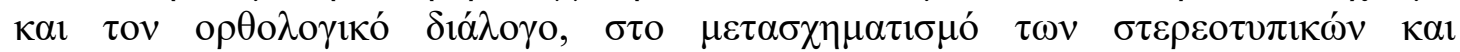

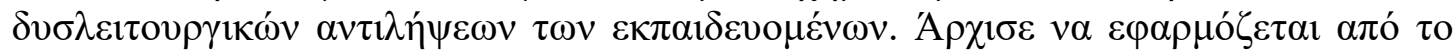

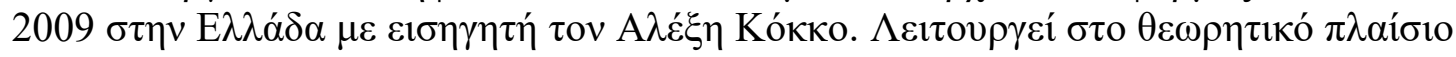

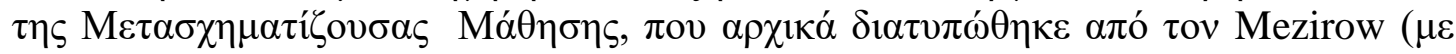

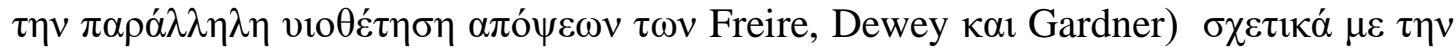

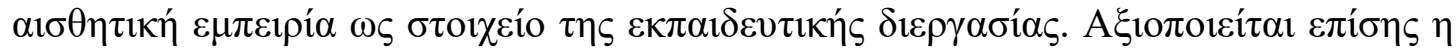

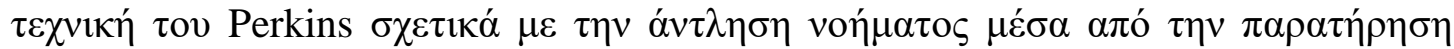
$\varepsilon \dot{\varepsilon} \gamma \omega \nu \tau \dot{\varepsilon} \chi v \eta \varsigma$.

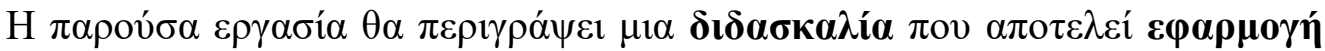

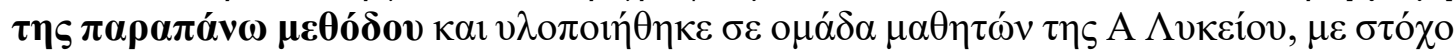

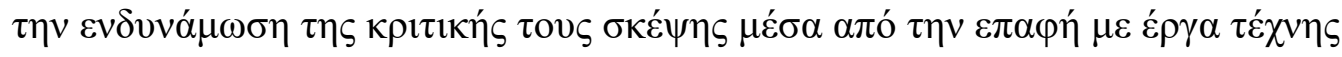

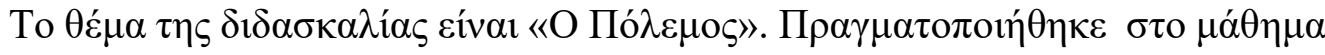

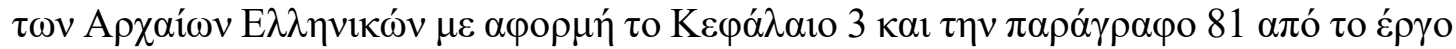

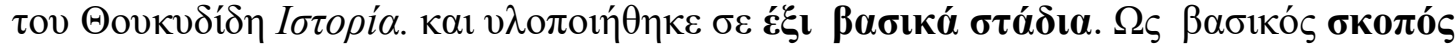

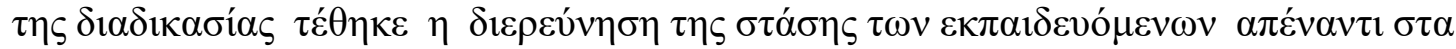

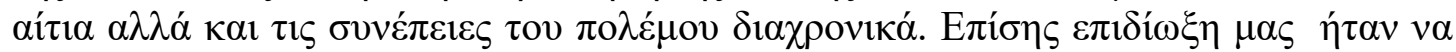

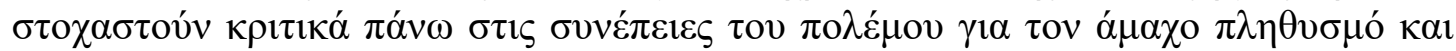

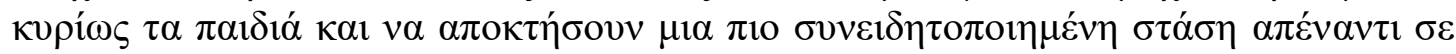

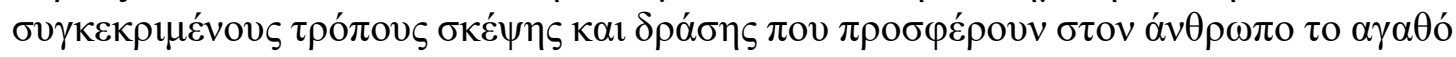

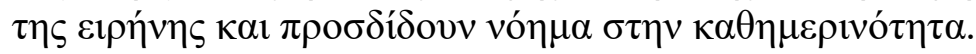

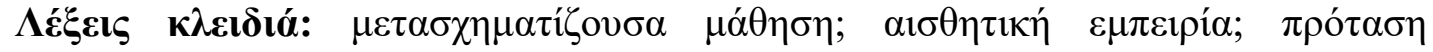
$\delta 1 \delta \alpha \sigma \kappa \alpha \lambda i ́ \alpha$.

\footnotetext{
Abstract

Transforming Learning through aesthetic experience involves a didactic and learning approach that aims, through critical thinking and rational dialogue, in transforming the stereotyped and dysfunctional perceptions of learners. It has been implemented since 2009 in Greece with Alexis Kokkos reporter. It works in the theoretical framework of Transforming Learning, originally formulated by Mezirow
} 
(while adopting Freire, Dewey and Gardner's views) on aesthetic experience as an element of the educational process. Perkins' technique of pumping meaning is also exploited through the observation of works of art.

The present work will describe a teaching that is an application of the above method and was implemented in a group of students of the 1st Lyceum, aiming at strengthening their critical thinking through contact with works of art

The subject of teaching is "The War". It was held in the class of ancient Greek on the occasion of Chapter 3 and paragraph 81 of Thucydides' work History and was implemented in six key stages. The main purpose of the process was to investigate the attitudes of learners towards the causes and consequences of the war over time.

Our aim was also to critically reflect on the consequences of the war for the civilian population and especially the children and to gain a more conscious attitude towards specific ways of thinking and acting that offer people the good of peace and give meaning to everyday life.

Key words: transforming learning; aesthetic experience; teaching suggestio

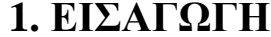

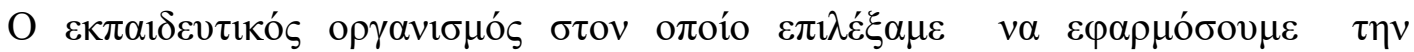

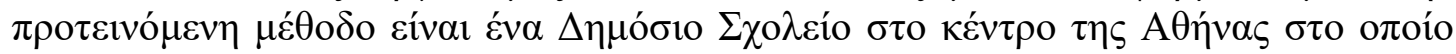

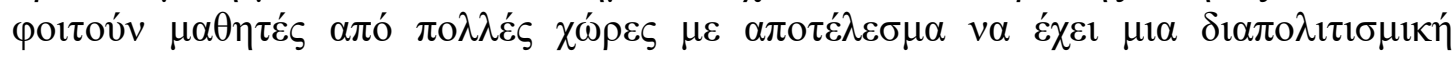

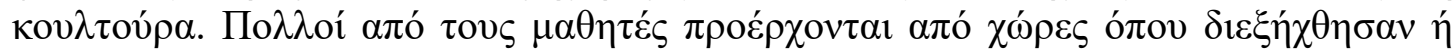

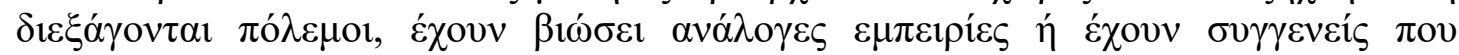

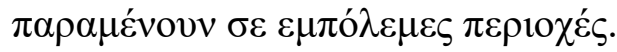

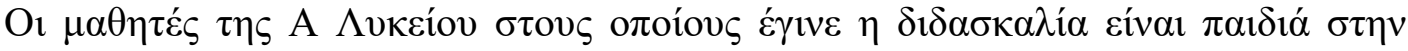

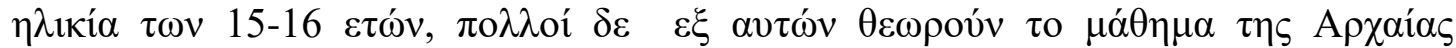

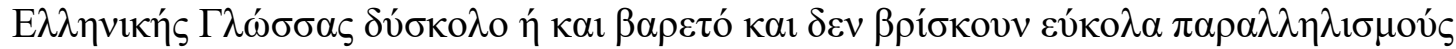

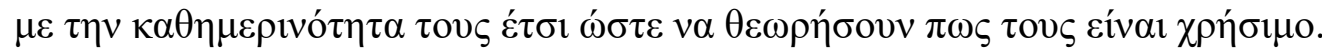

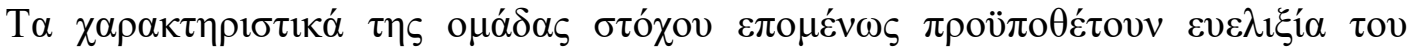

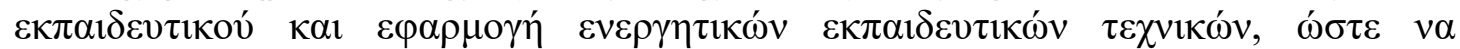

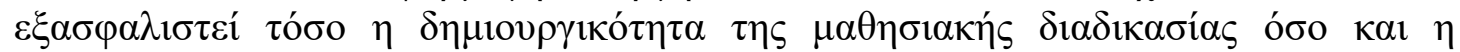

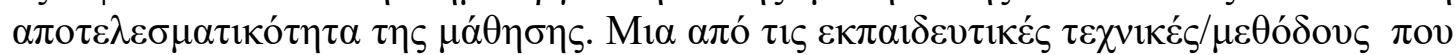

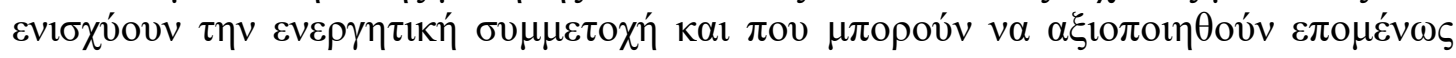

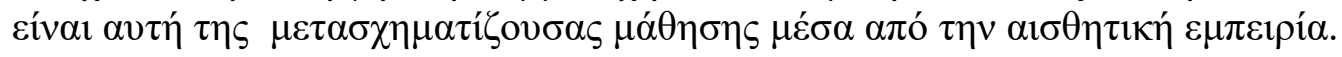

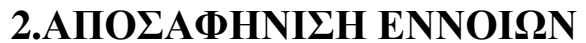

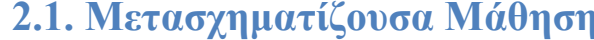

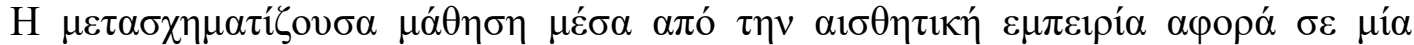

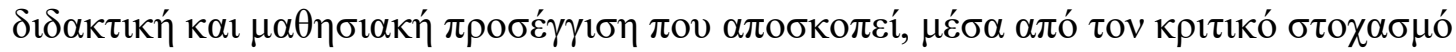

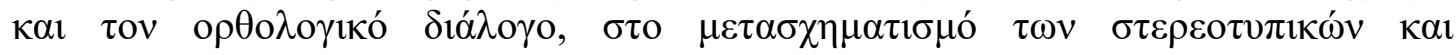

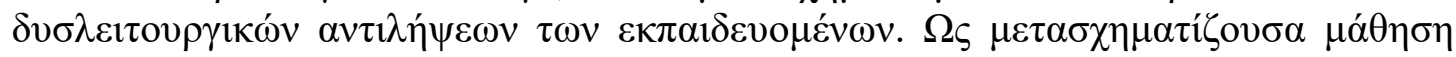

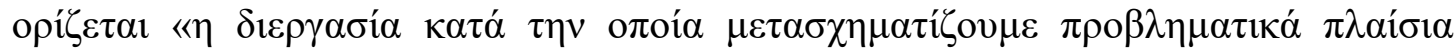

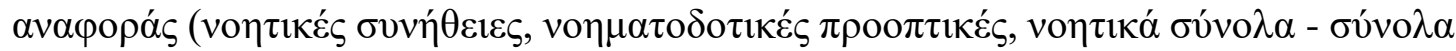

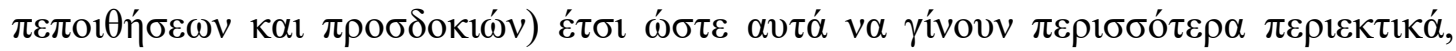




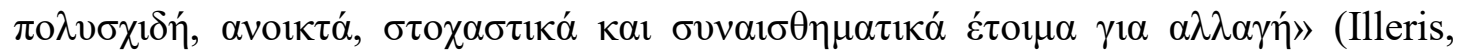
2009).

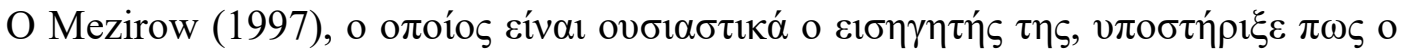

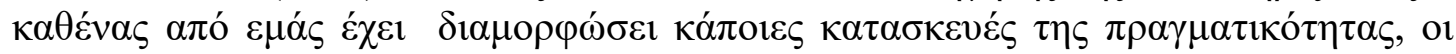

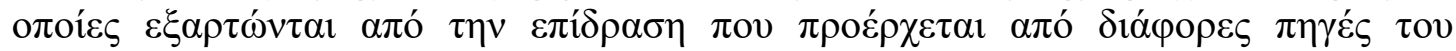

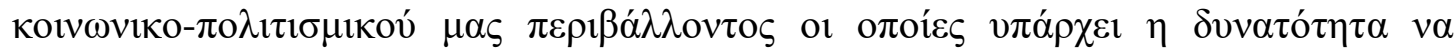

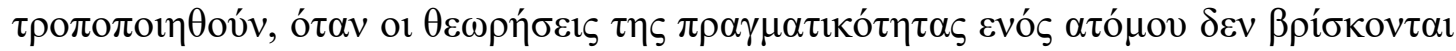

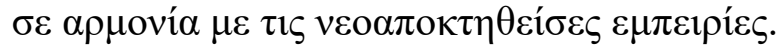

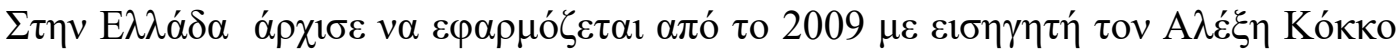

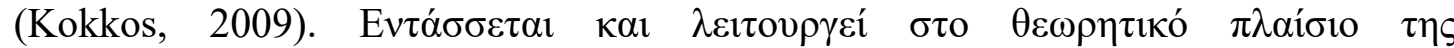

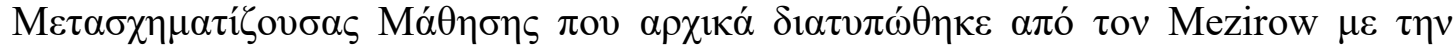

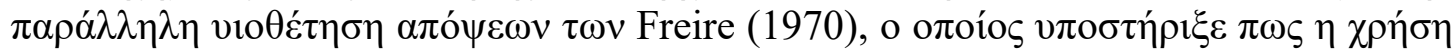

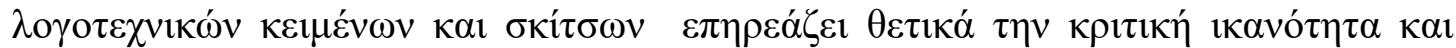

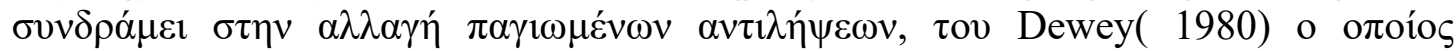

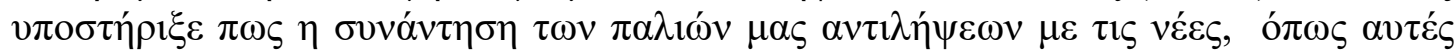

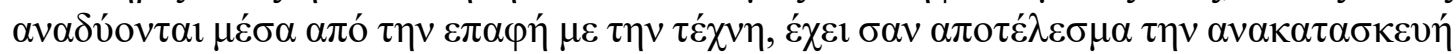

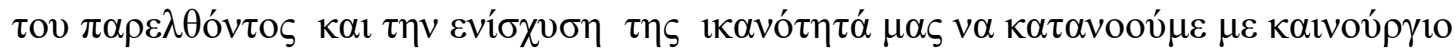

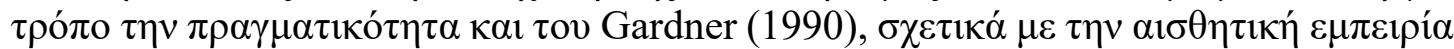

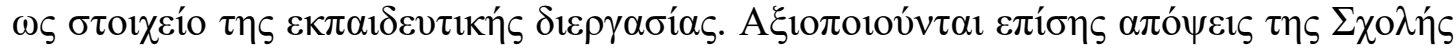

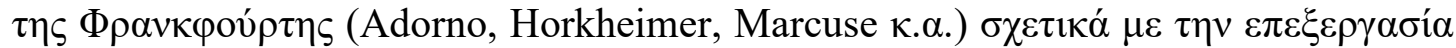

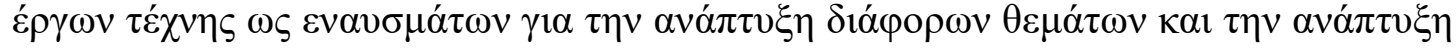

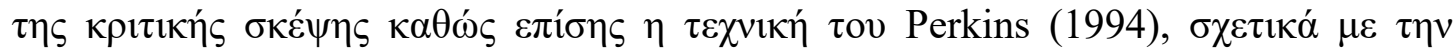

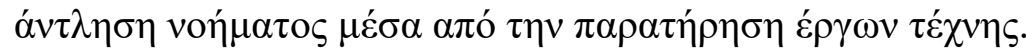

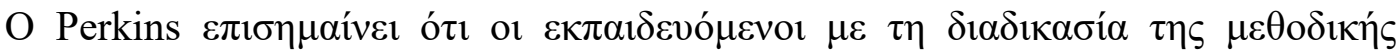

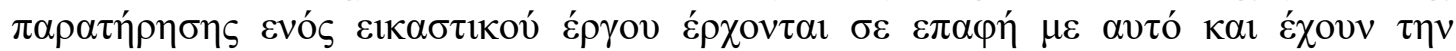

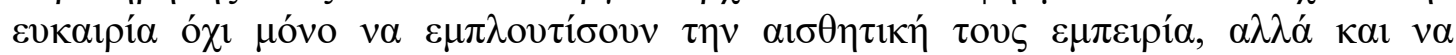

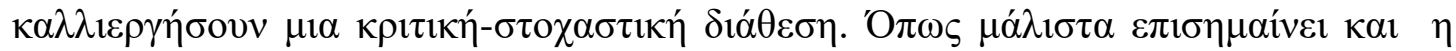

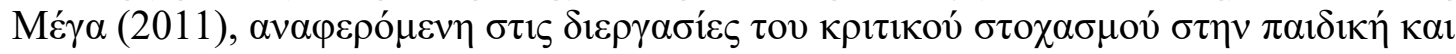

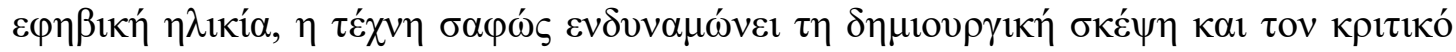

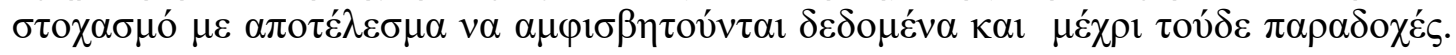

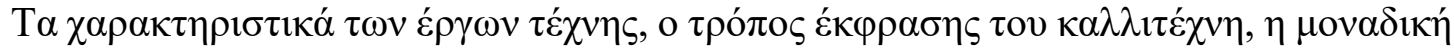

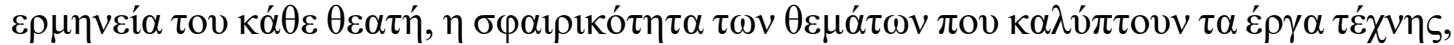

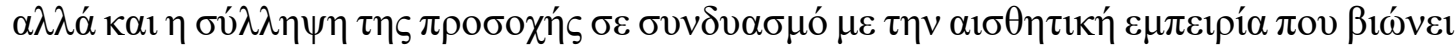

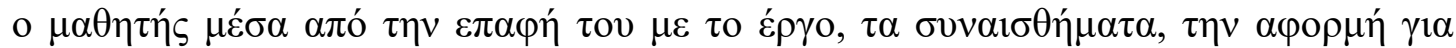

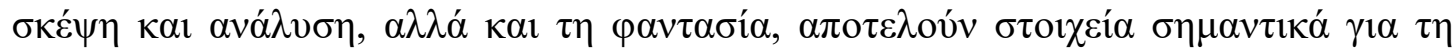

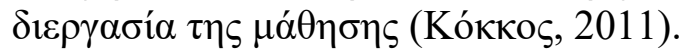

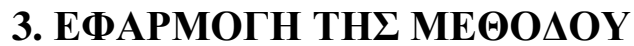

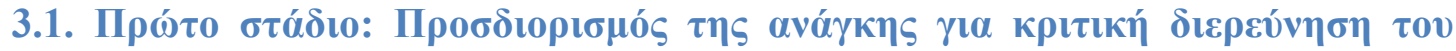 $\theta \dot{\varepsilon} \mu \alpha \tau 0 \varsigma$}

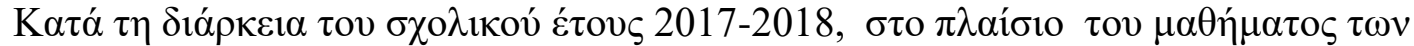

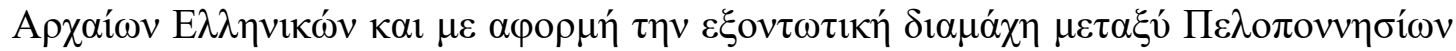

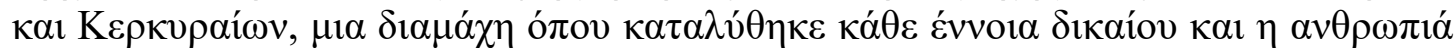
$\pi \alpha \rho \alpha \gamma v \omega \rho i ́ \sigma \theta \eta \kappa \varepsilon \varepsilon v \tau \varepsilon \lambda \omega ́$ s. 


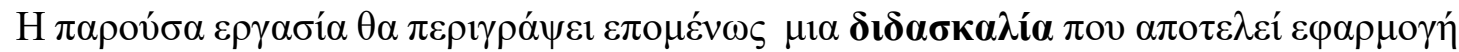

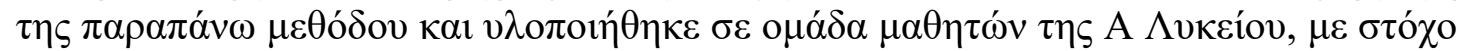

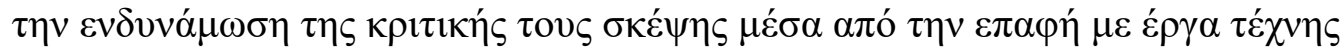

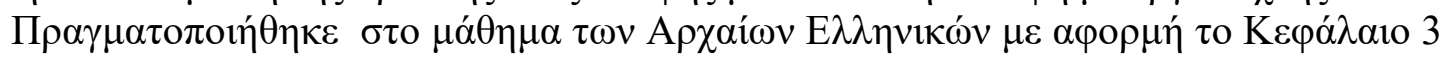

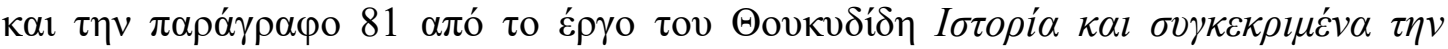

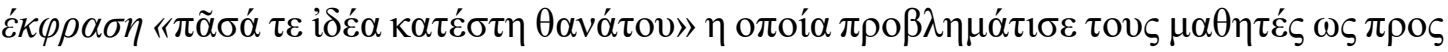

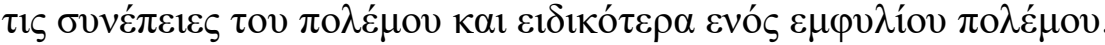

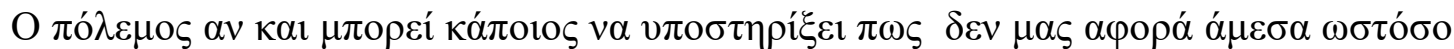

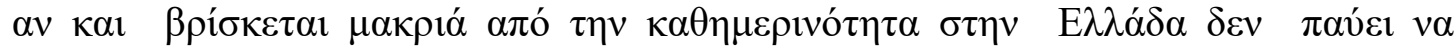

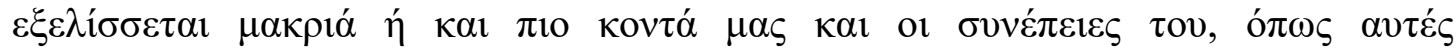

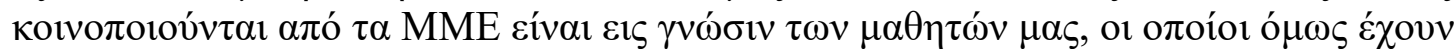

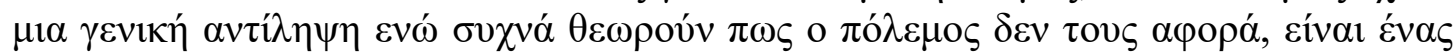

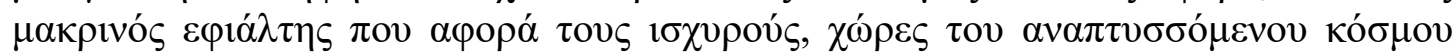

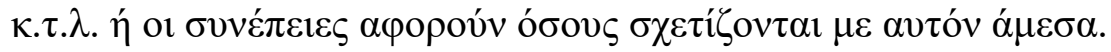

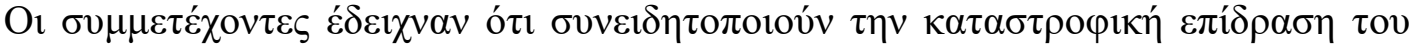

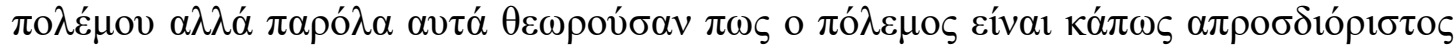

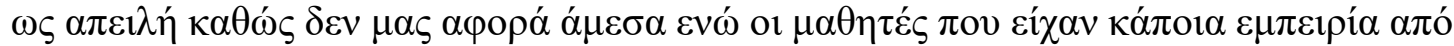

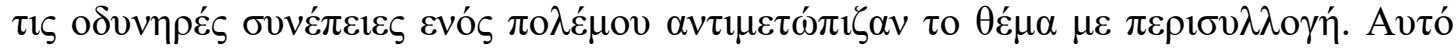

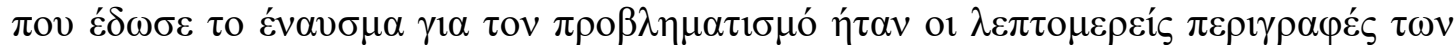

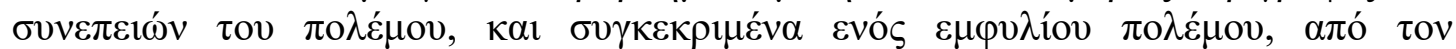

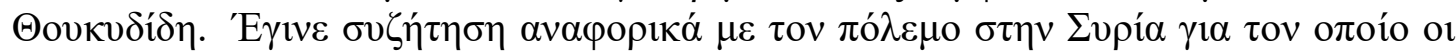

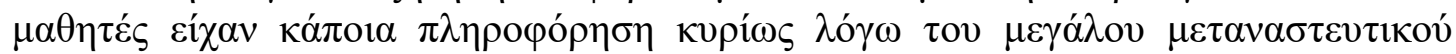

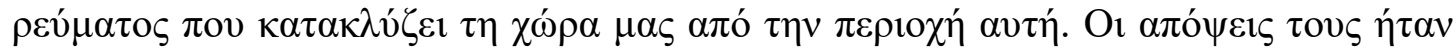

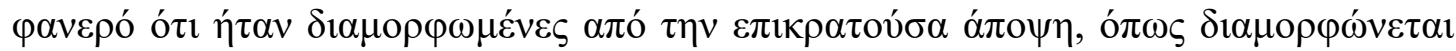

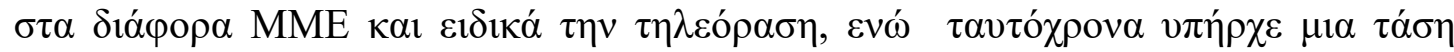

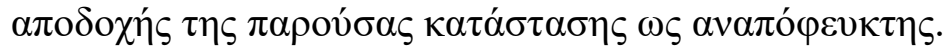

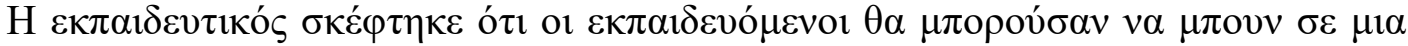

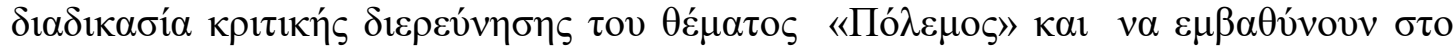

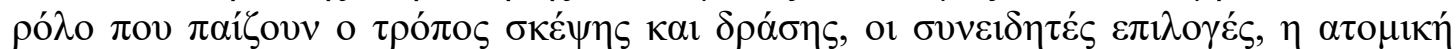

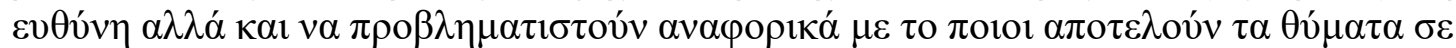

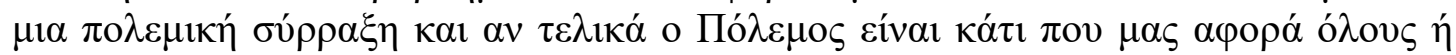

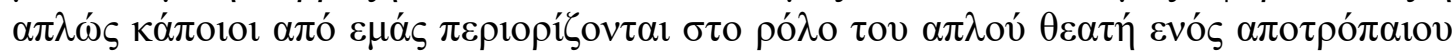

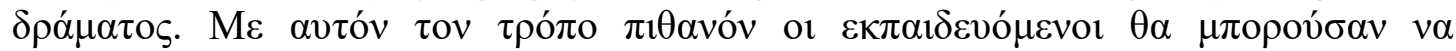

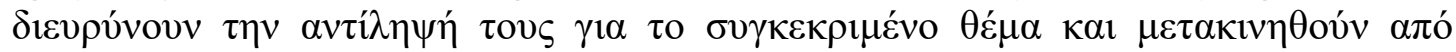

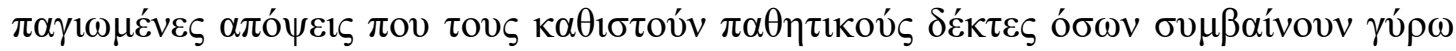
tovs.

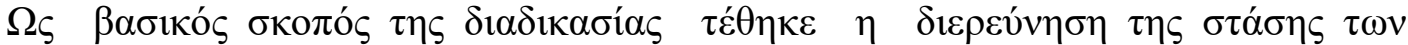

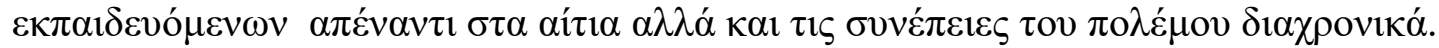

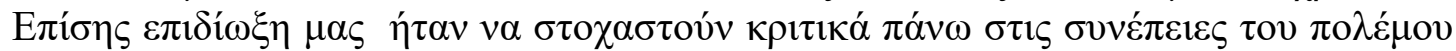

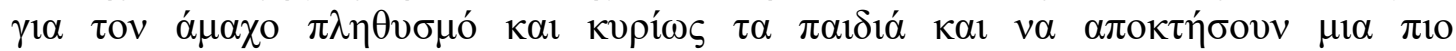

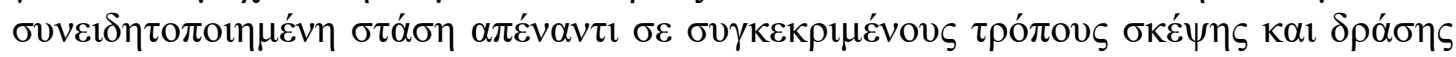

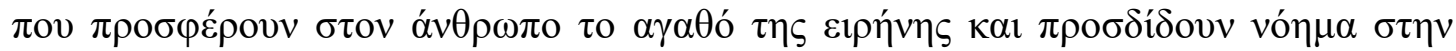

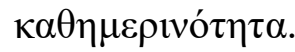

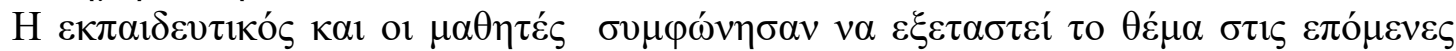

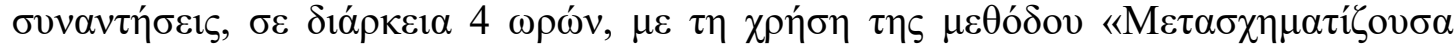

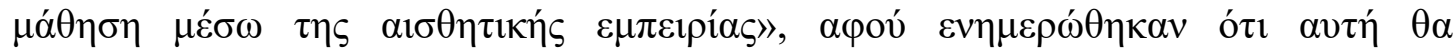




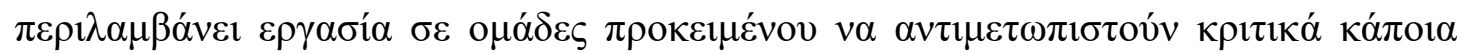

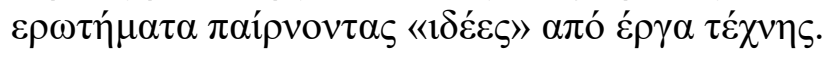

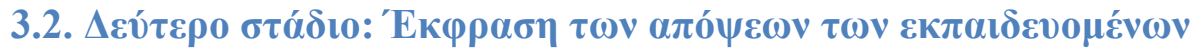

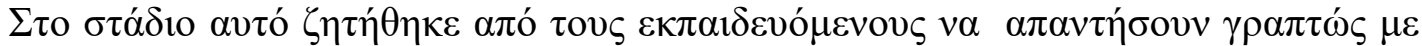

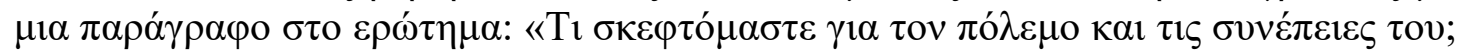

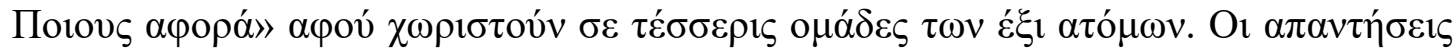

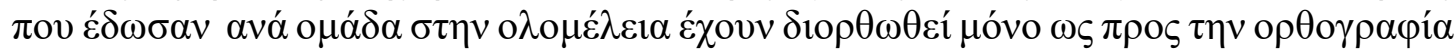

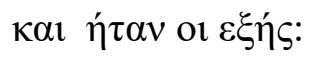

O $\mu \alpha ́ \delta \alpha 1 \eta$

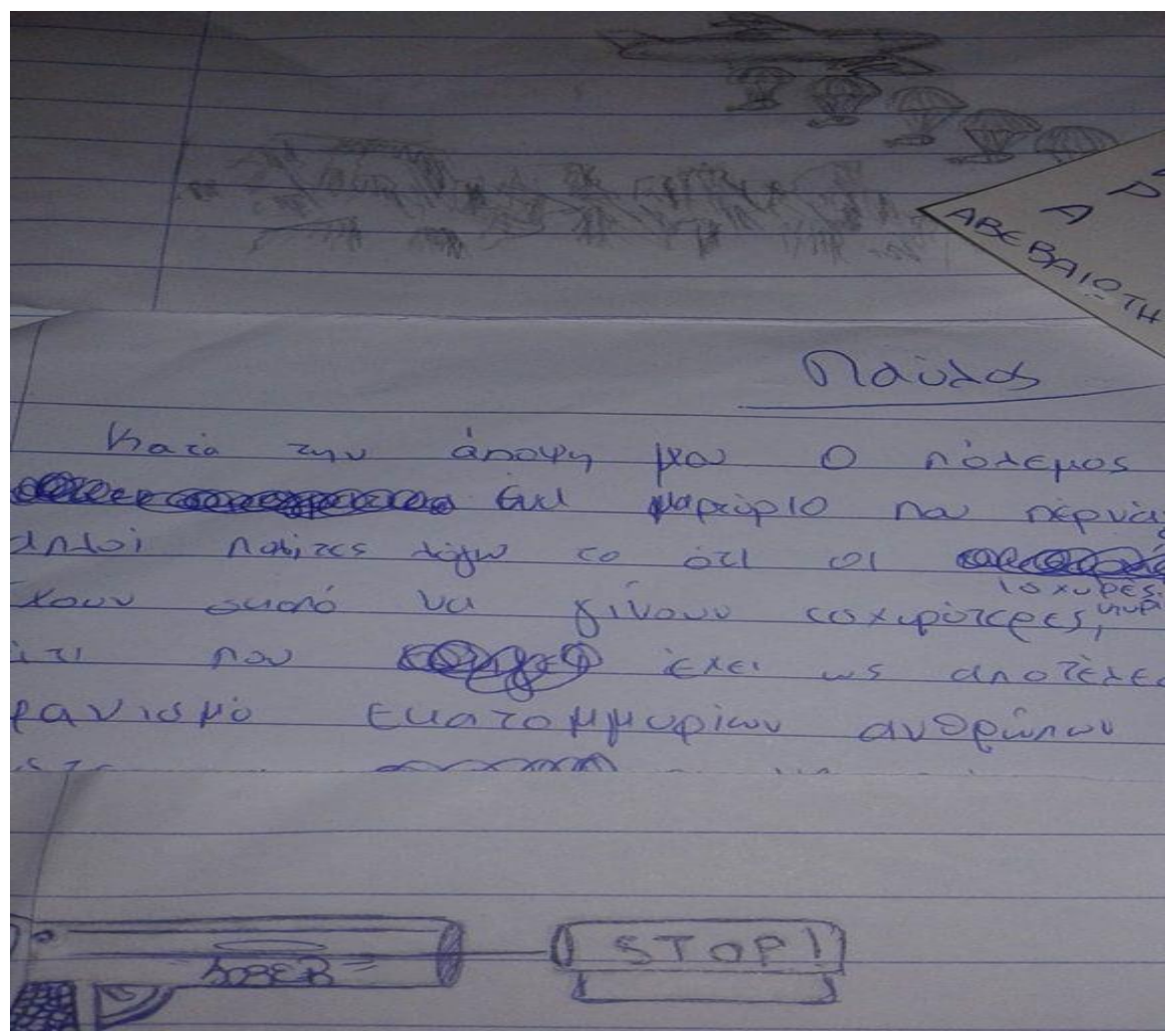

$\mathrm{O} \mu \alpha \dot{\delta} \delta \alpha 2^{\eta}$ 


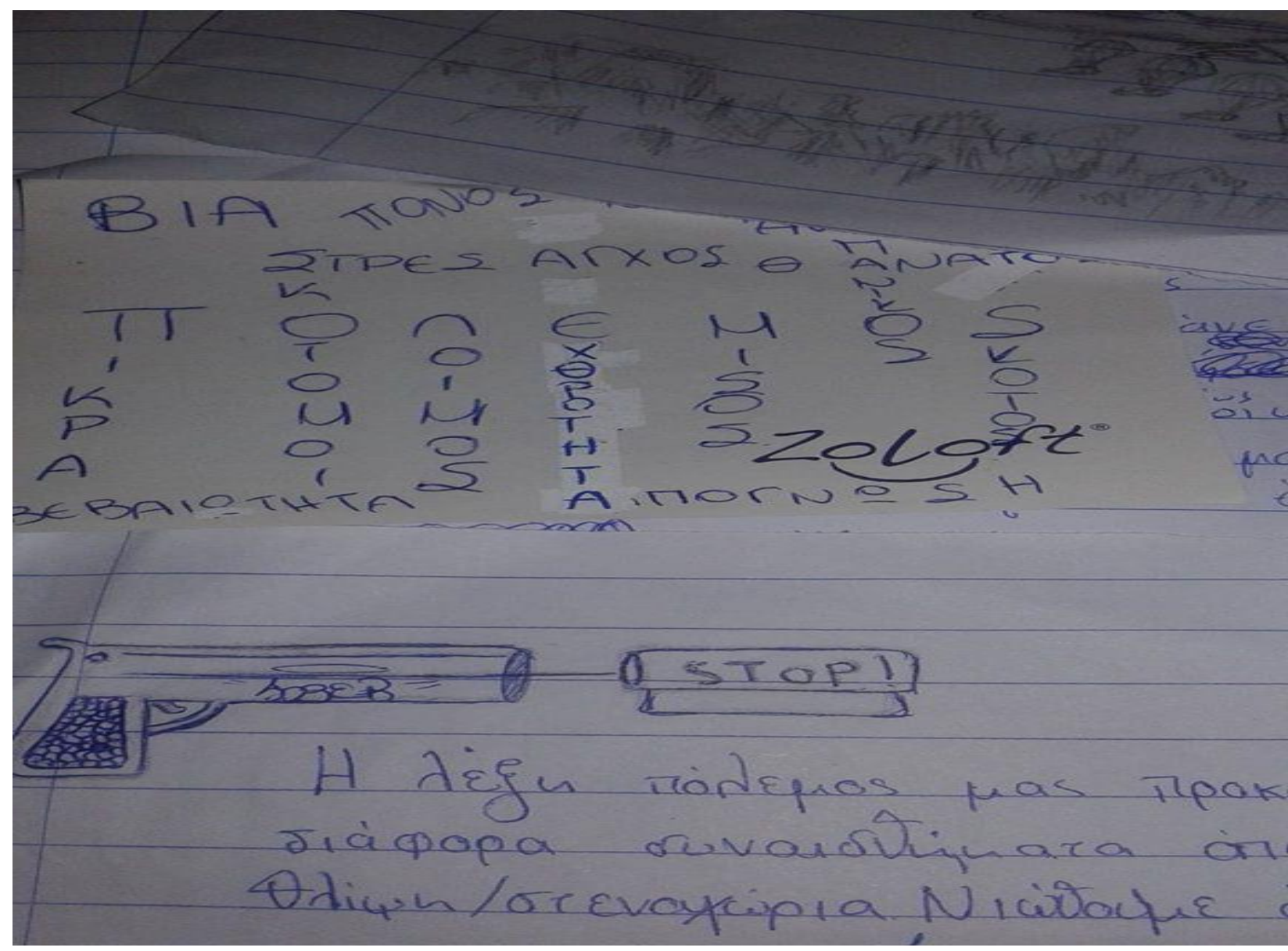

O $\mu \alpha \dot{\delta} \delta 3^{\eta}$

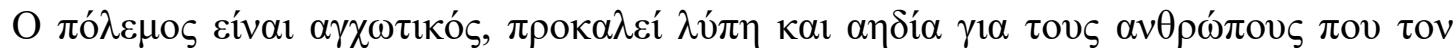

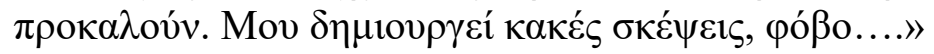

O $\mu \alpha \dot{\delta} \delta 4^{\eta}$

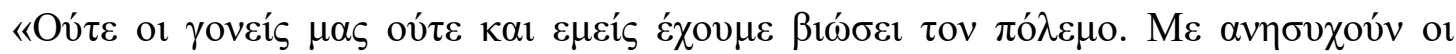

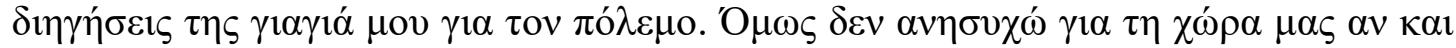

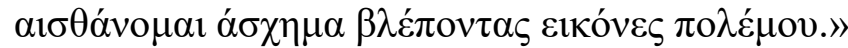

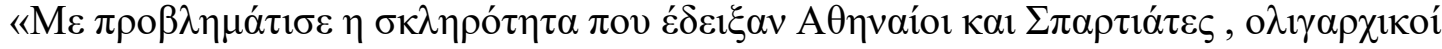

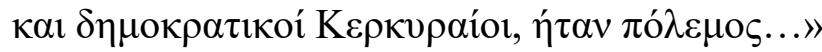

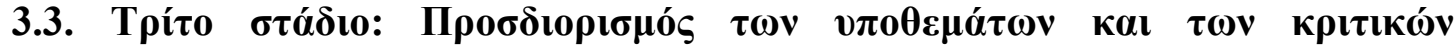 $\varepsilon \rho \omega \tau \eta \mu \alpha ́ \tau \omega v$}

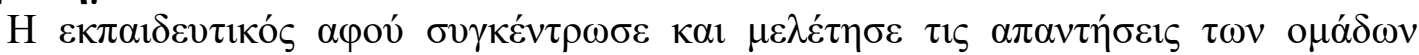

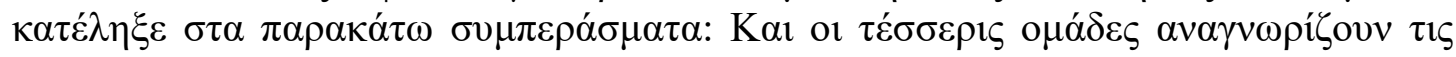

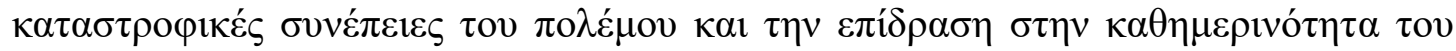

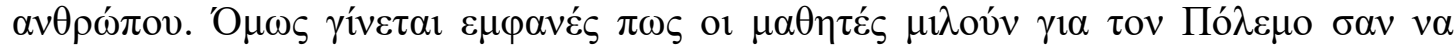

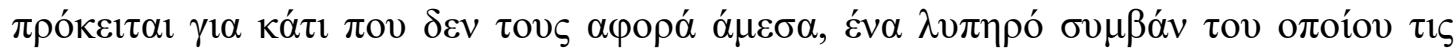

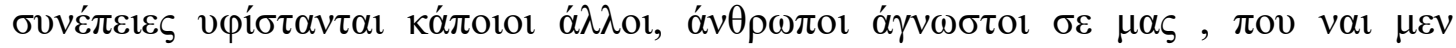




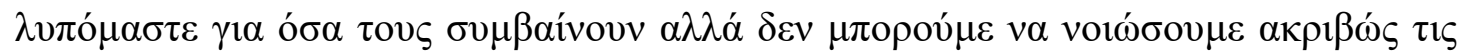

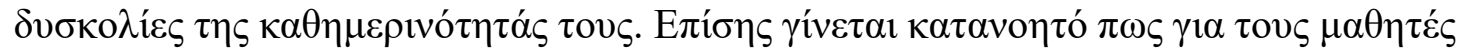

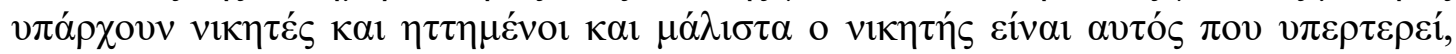

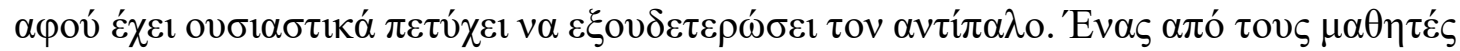

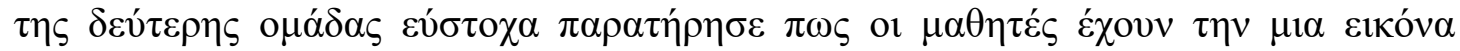

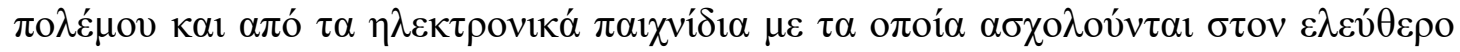

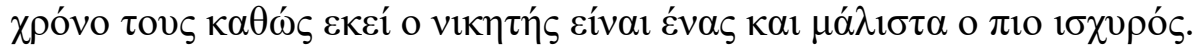

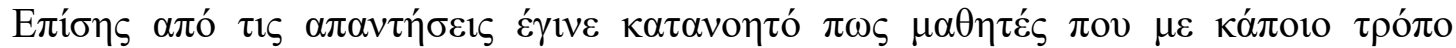

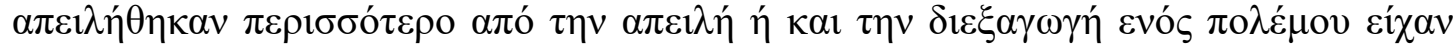

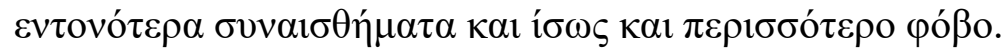

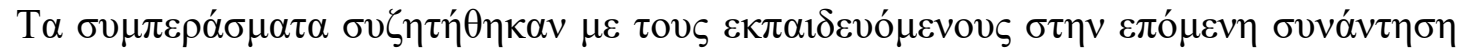

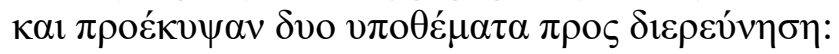

A)H $\varepsilon \pi 1 \delta i ́ \omega \xi \eta \tau \eta \varsigma \varepsilon v \tau v \chi i ́ \alpha \varsigma \alpha \pi o ́ ~ \tau o v ~ \alpha ́ v \theta \rho \omega \pi \mathrm{o}$.

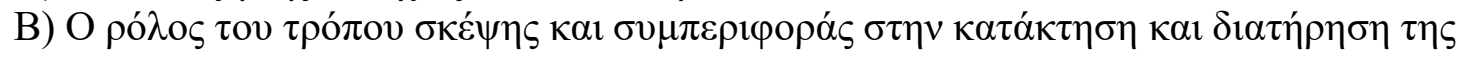

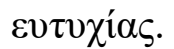

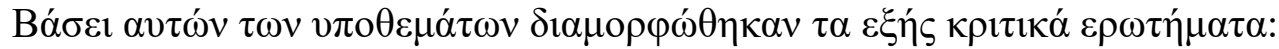

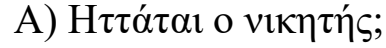

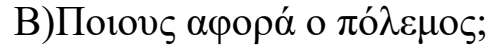

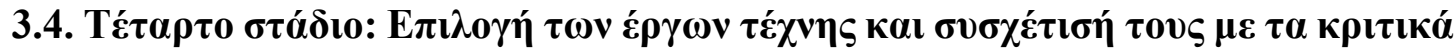 $\varepsilon \rho \omega \tau \eta ́ n \mu \tau \alpha$}

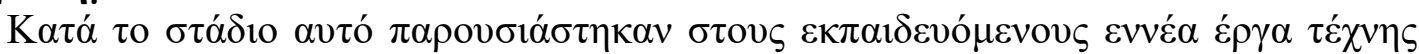

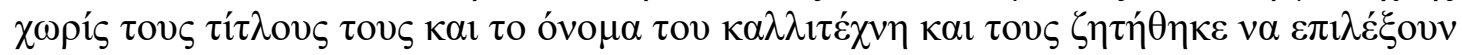

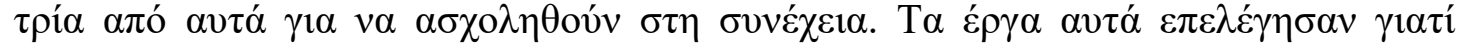

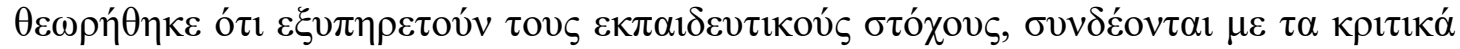

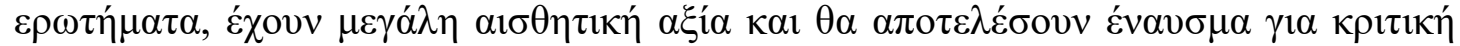

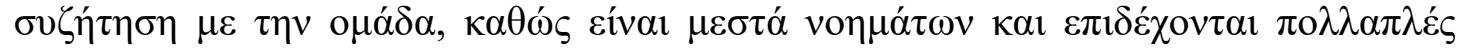

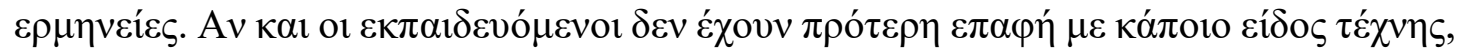

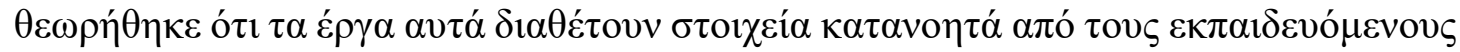

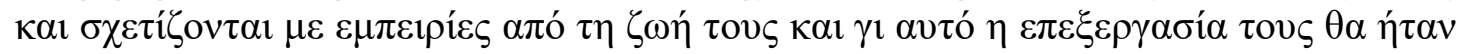

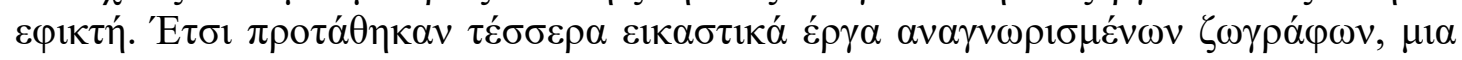

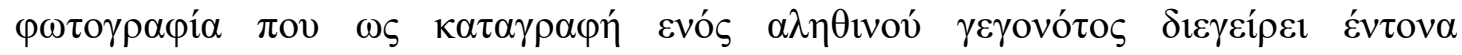

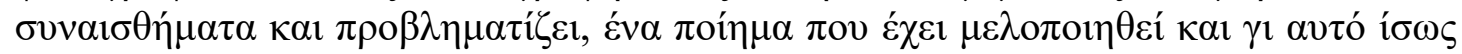

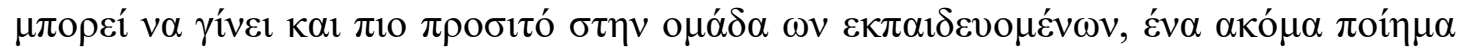

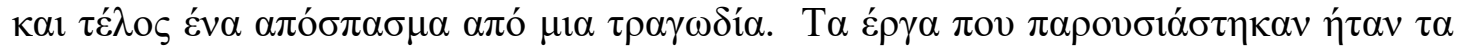
$\varepsilon \xi \dot{n} s:$

The Face of the War- Salvador Dali (1940)

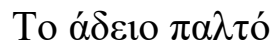

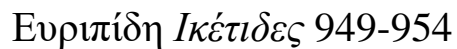

War- Marc Chagall (1966)

War News- Louisa Starr Canziani, 1900

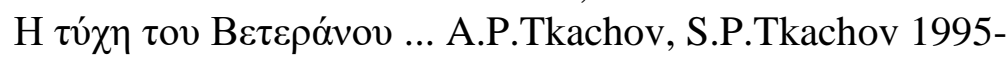

O Пó $\lambda \varepsilon \mu \circ \varsigma^{\prime \prime} \tau o v$ 'O $\tau \tau \circ \mathrm{N} \tau \imath \xi$

"The day the war came" $\tau\rceil \varsigma$ Nicola Davies

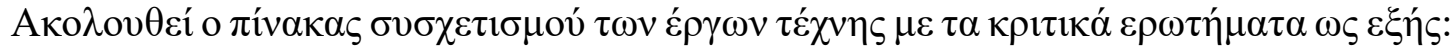




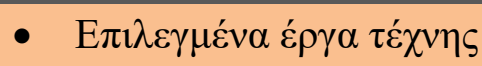

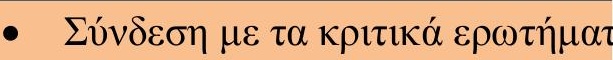

- A

- The Face of the War-Salvador Dali (1940)

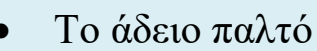

- Evpı

- War- Marc Chagall (1966)

- War News- Louisa Starr Canziani, 1900

- H $\mu$ śpa $\quad \pi 0 v \quad$ o ("The day the war came" $\tau\rceil \varsigma$ Nicola Davies)

)




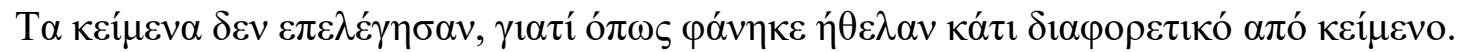

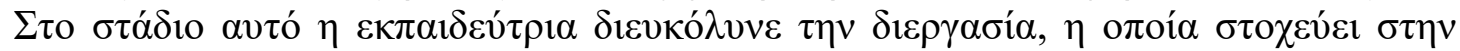

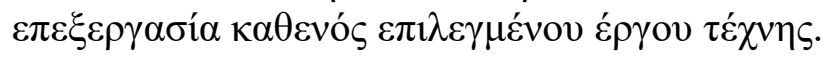

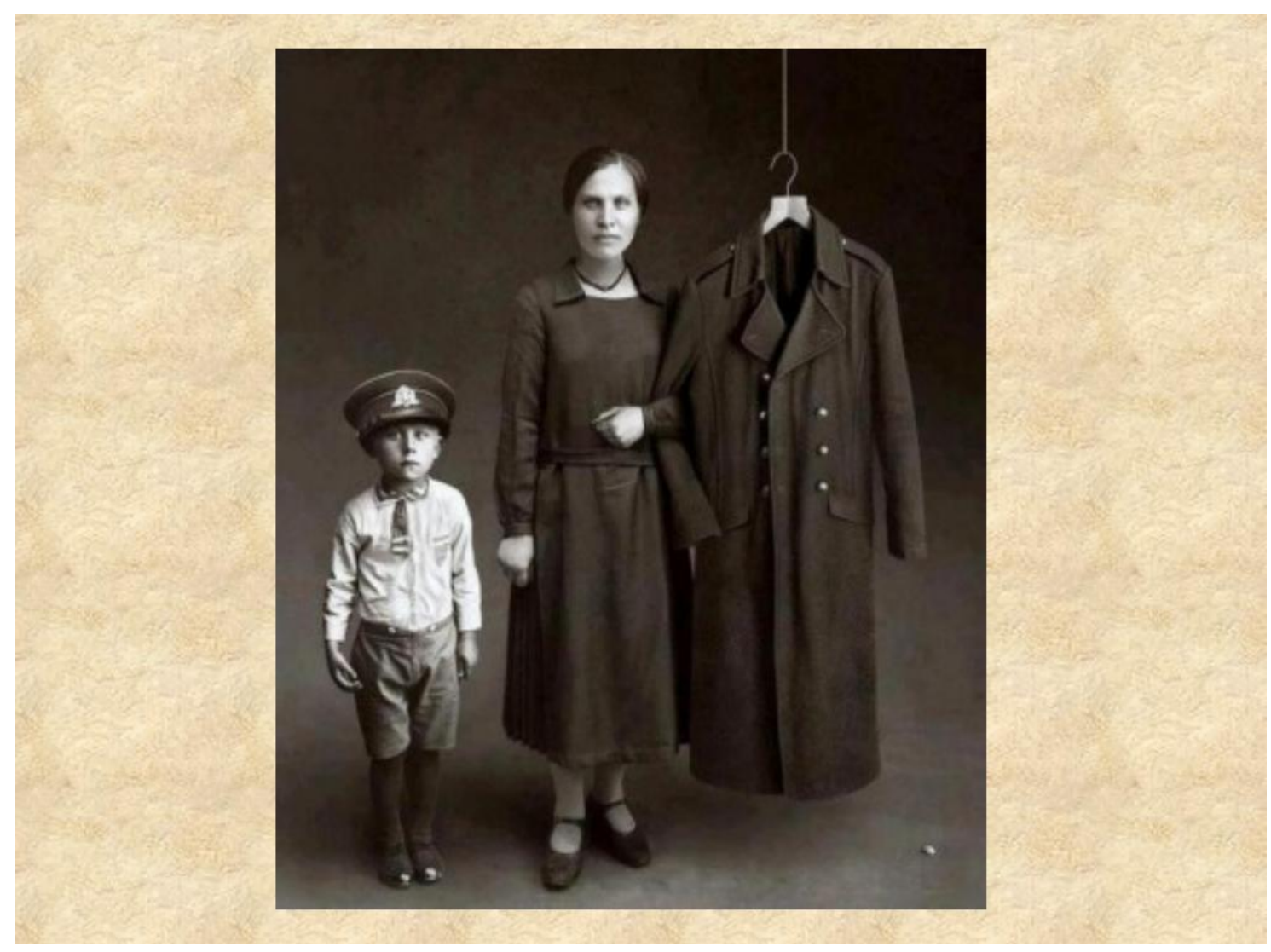

Eıкóva 1 

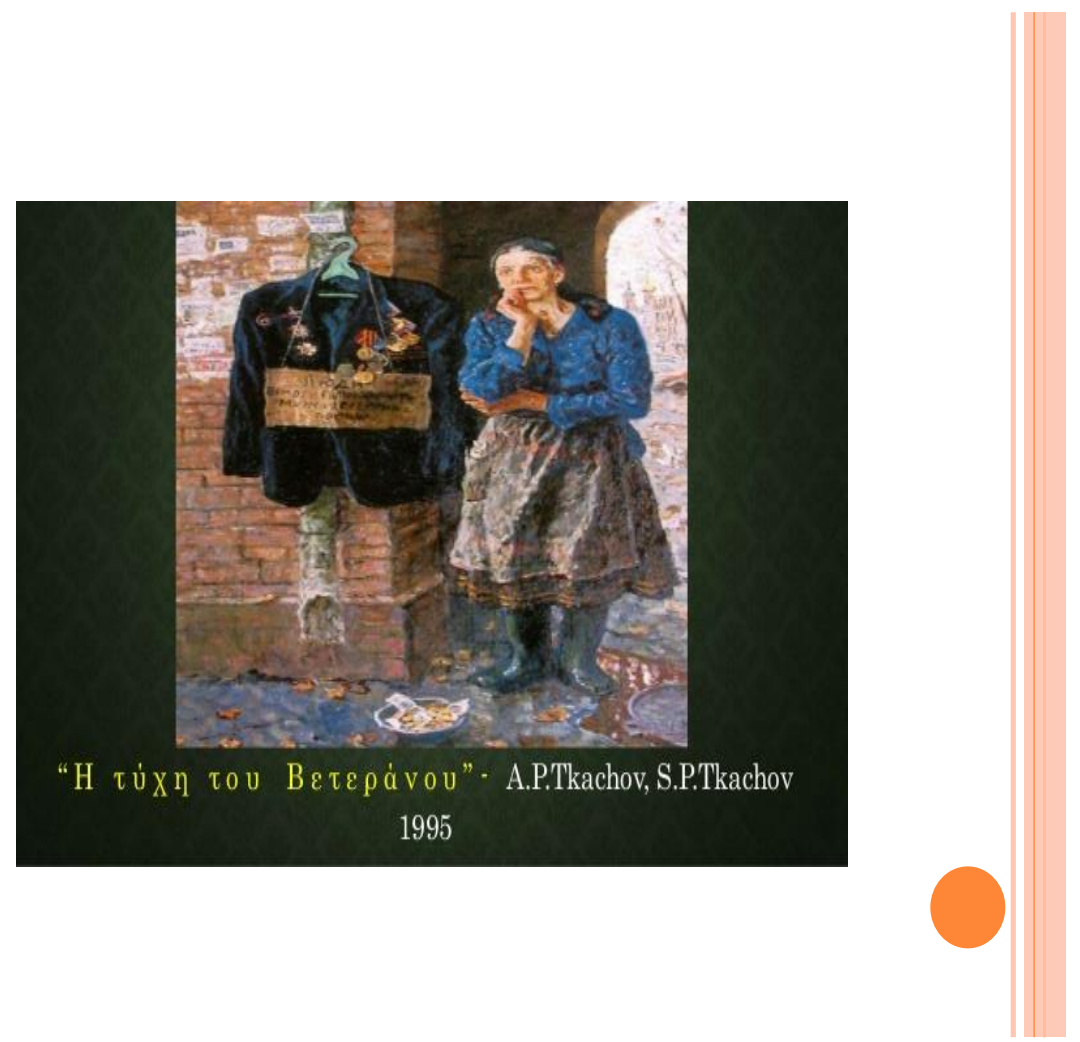

Eıкóva 2

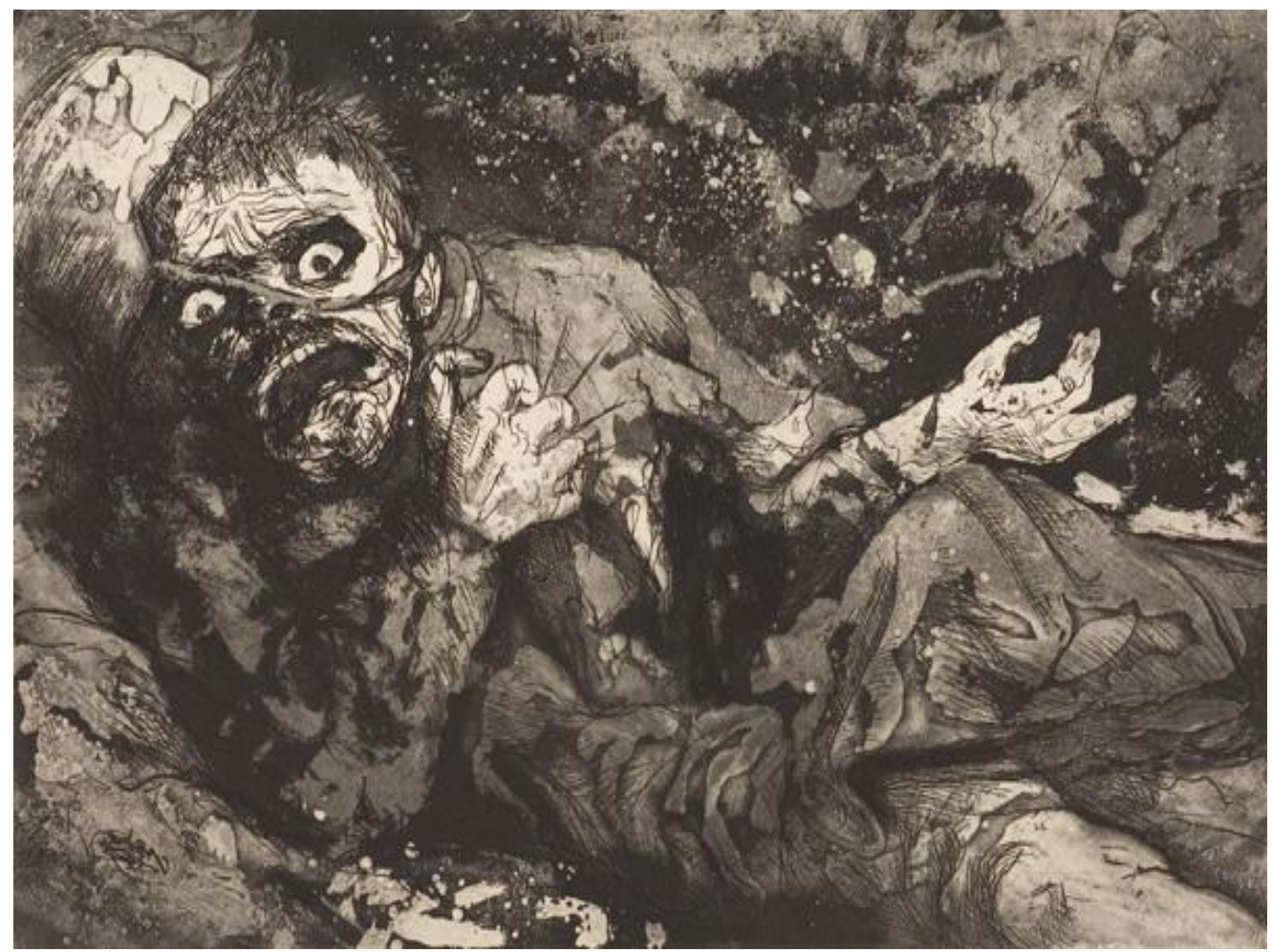

Eıкóva 3

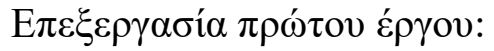




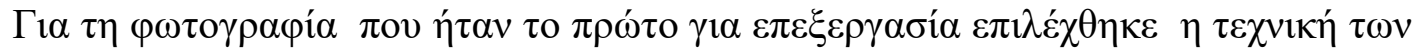

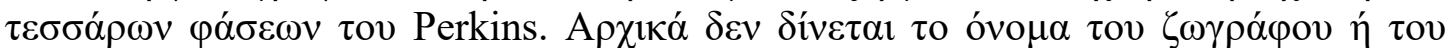

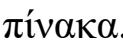

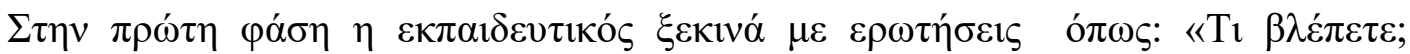

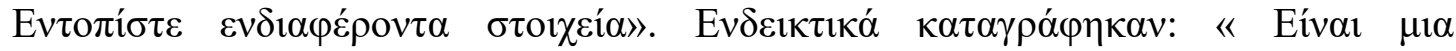

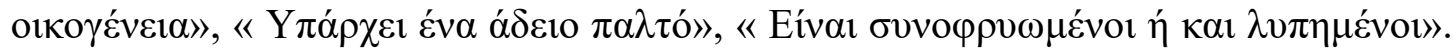

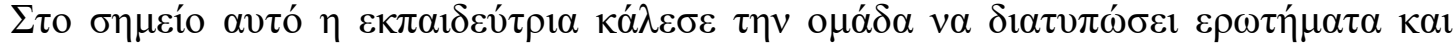

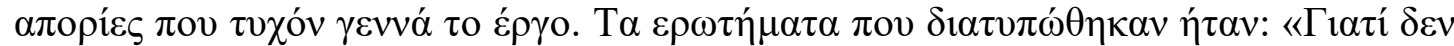

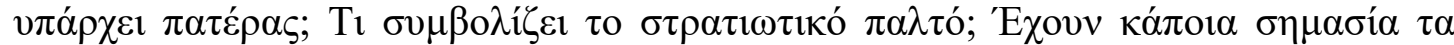

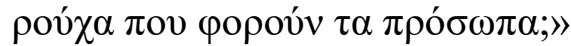

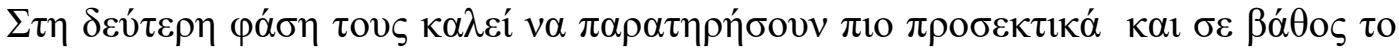

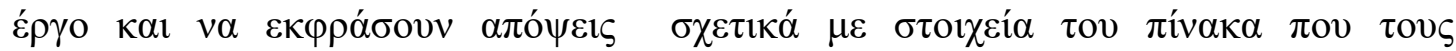

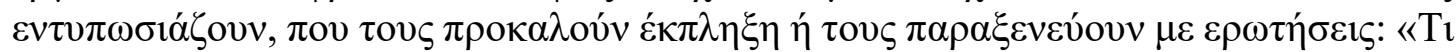

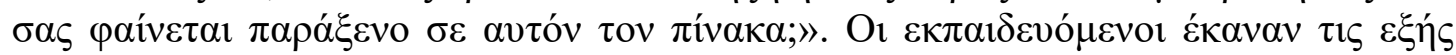

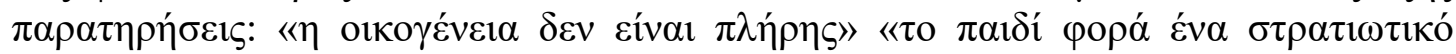

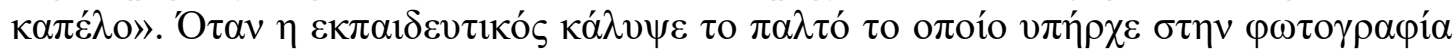

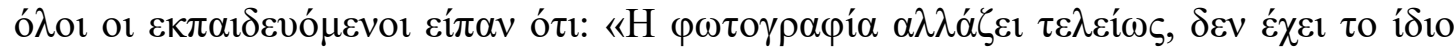

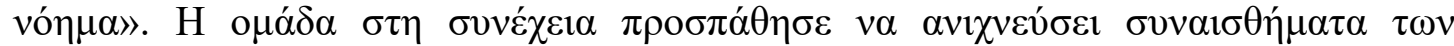

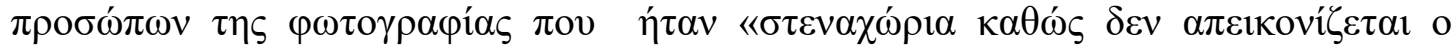

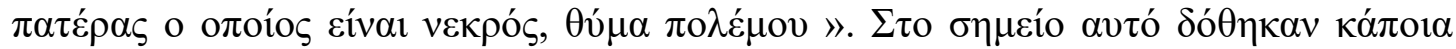

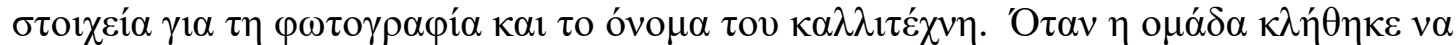

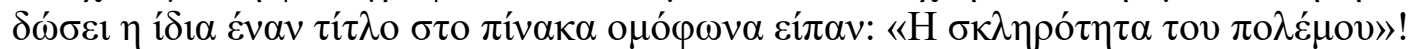

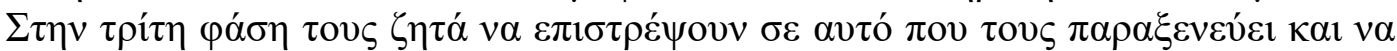

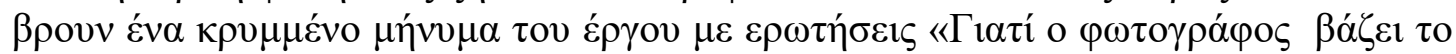

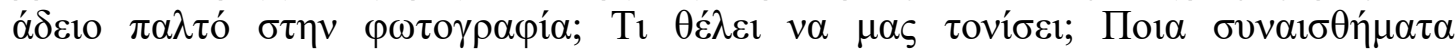

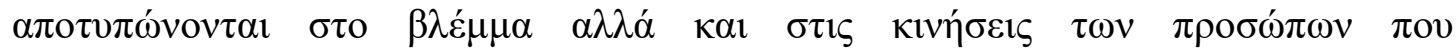

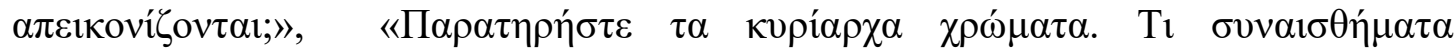

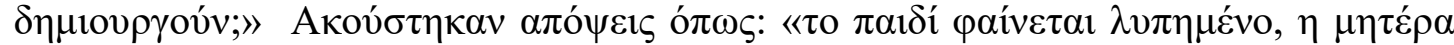

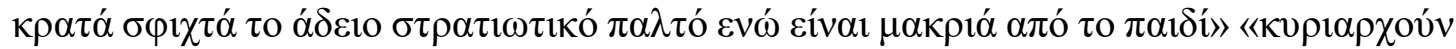

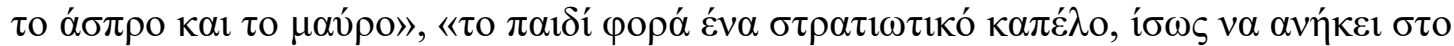

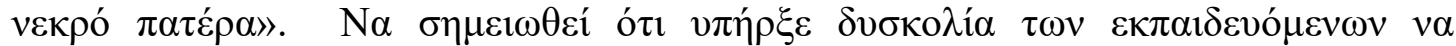

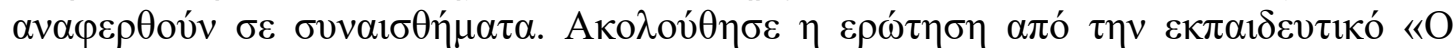

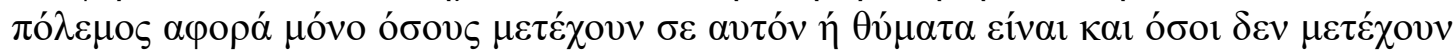

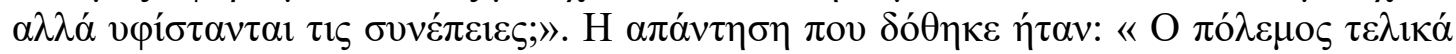

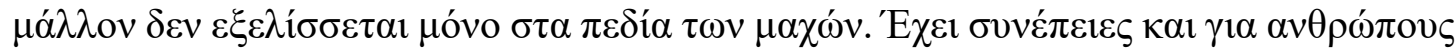

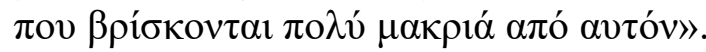

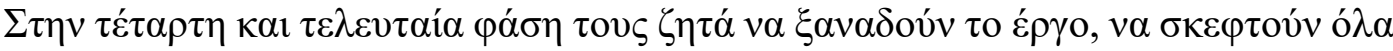

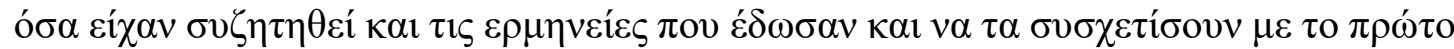

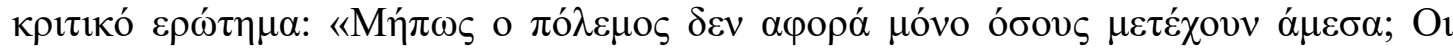

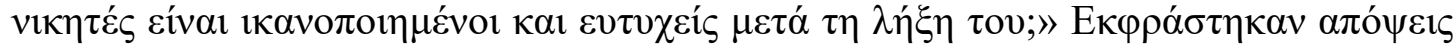

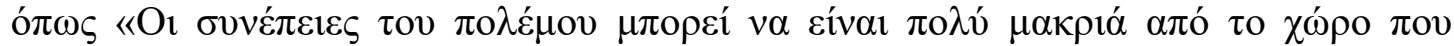

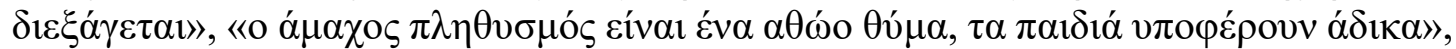

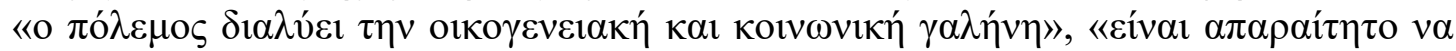

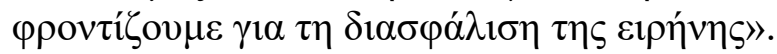

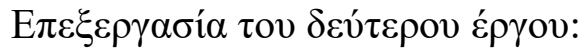

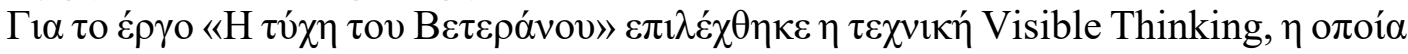

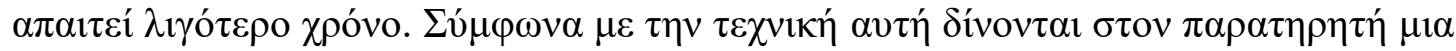

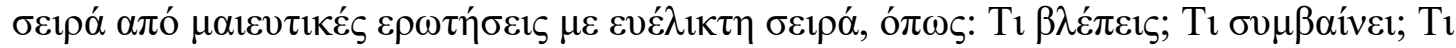

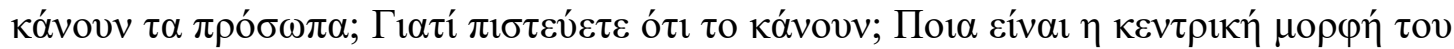




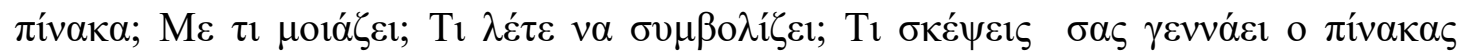

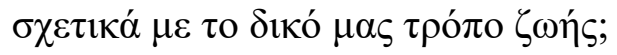

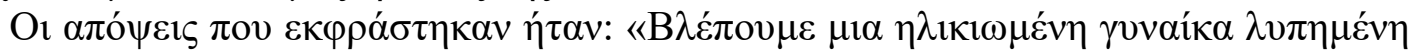

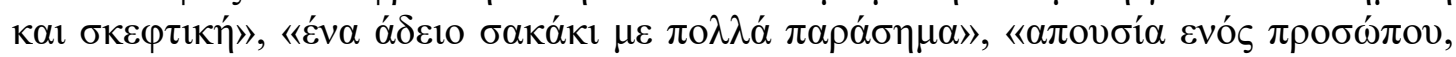

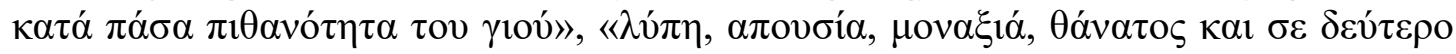

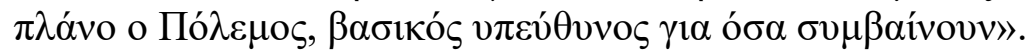

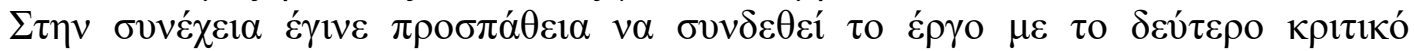

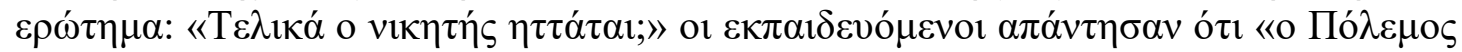

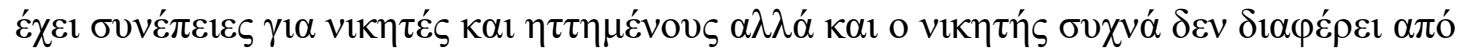

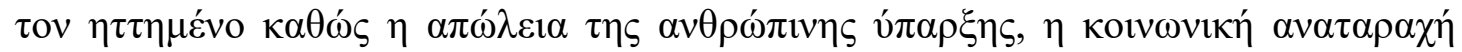

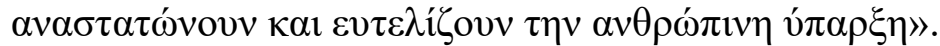

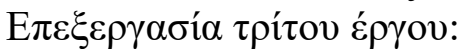

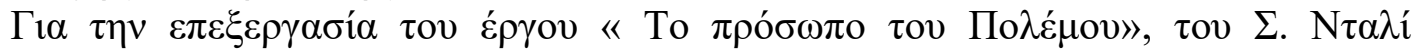

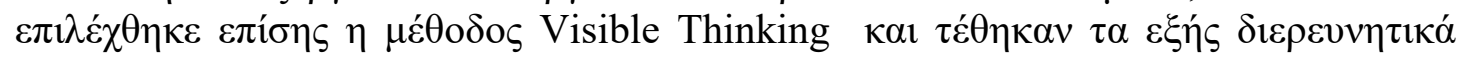

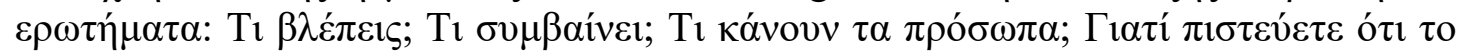

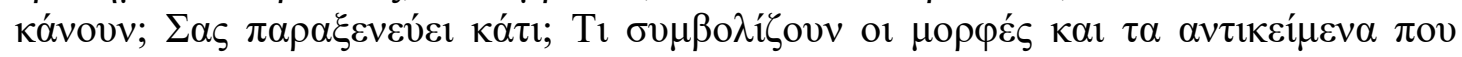

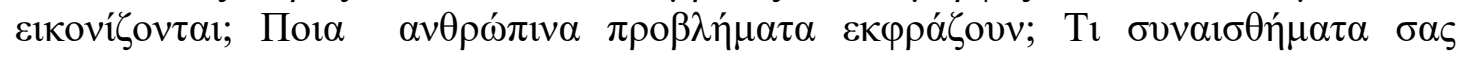

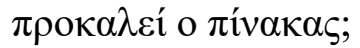

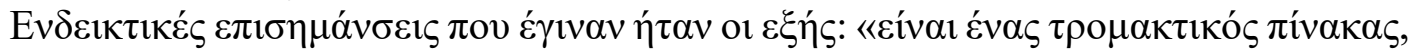

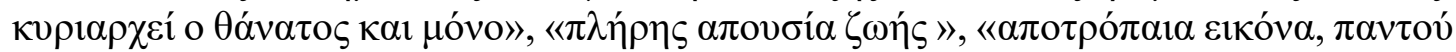

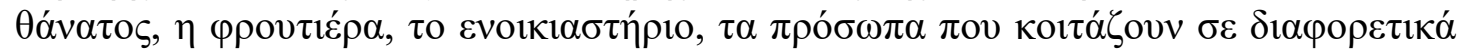

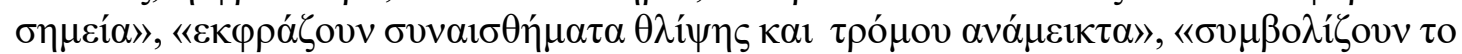

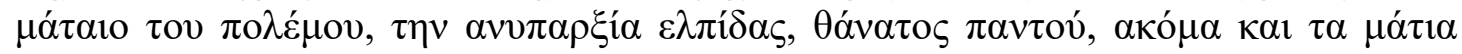

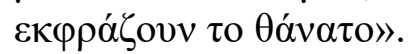

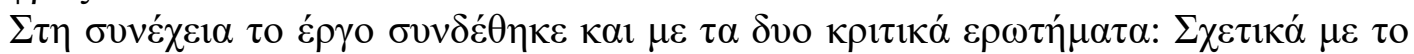

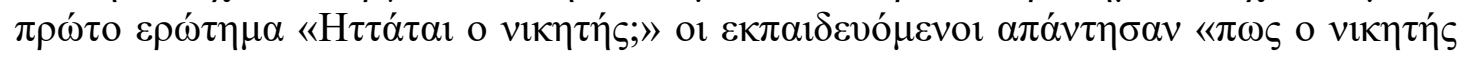

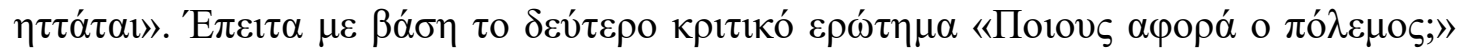

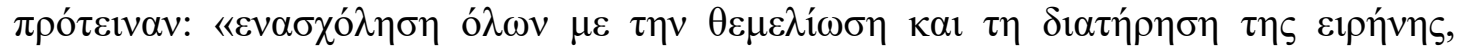

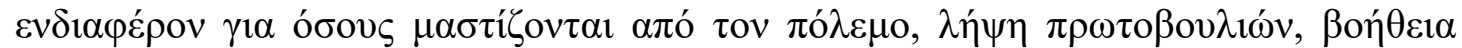

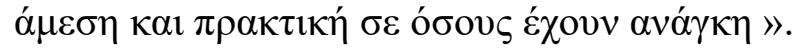

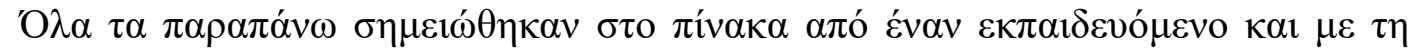

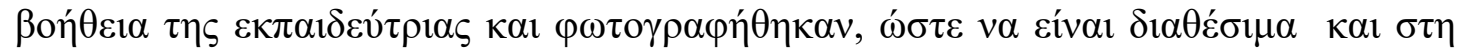

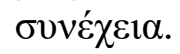

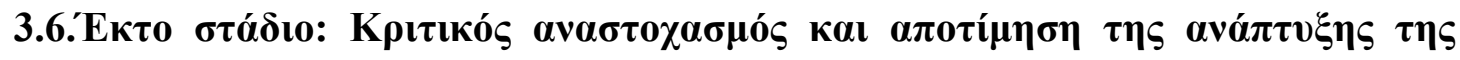

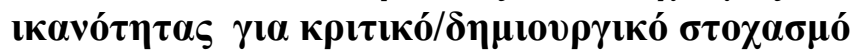

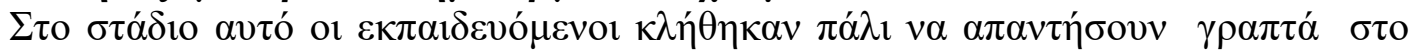

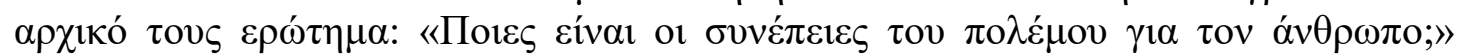

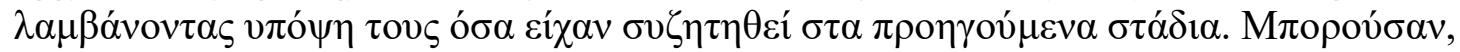

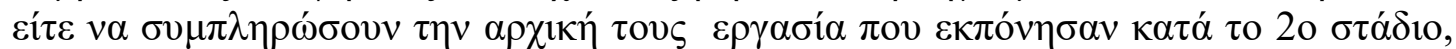

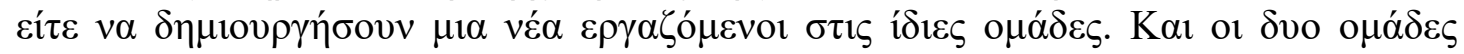

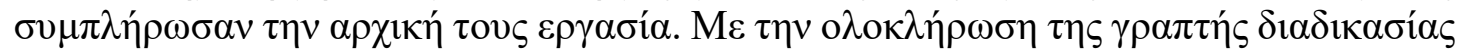

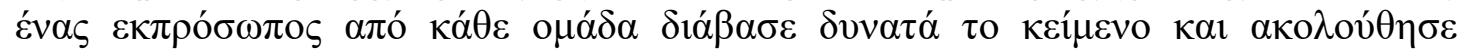

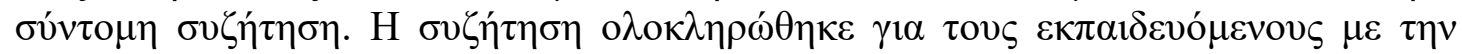

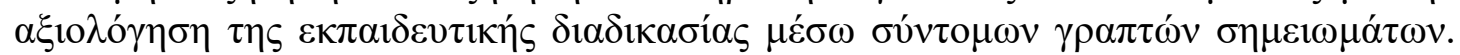

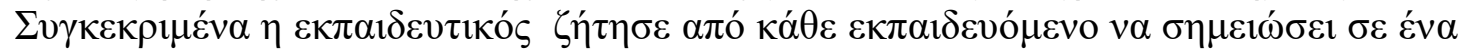

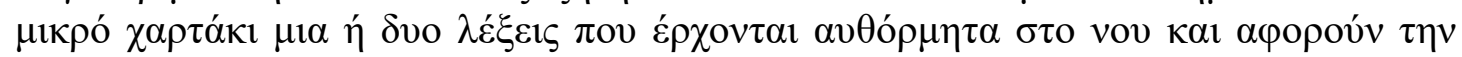

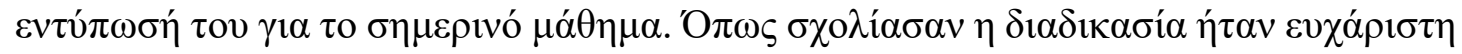
$\kappa \alpha l \varepsilon v \delta 1 \alpha \varphi \varepsilon ́ \rho o v \sigma \alpha$. 


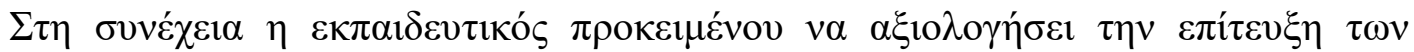

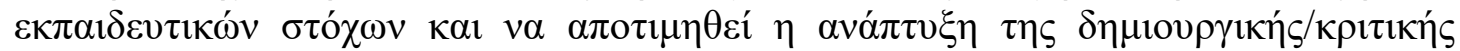

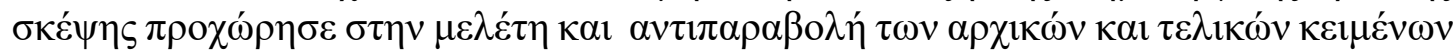

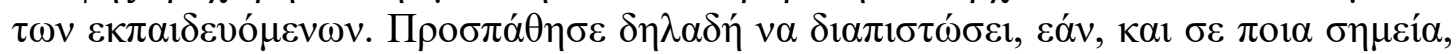

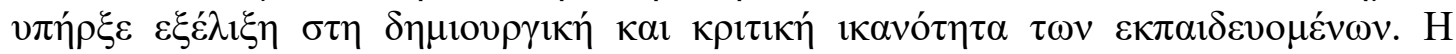

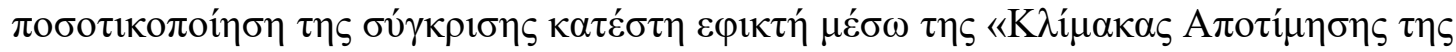

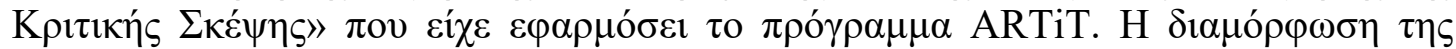

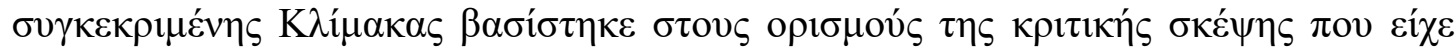

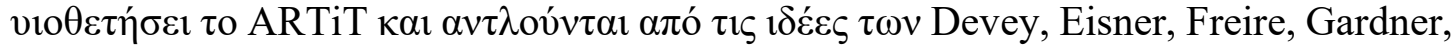
Mezirow, Brookfield $\kappa \alpha$ I Perkins.

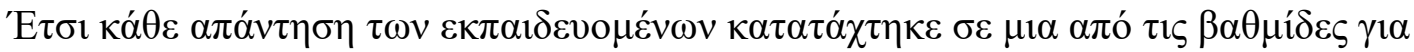

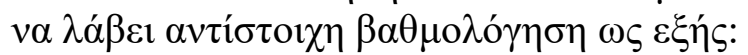

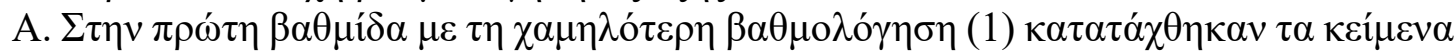

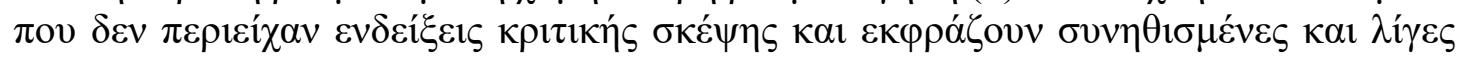
$1 \delta \varepsilon \dot{\varepsilon} \varsigma$.

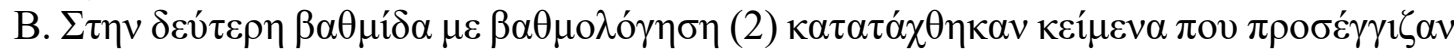

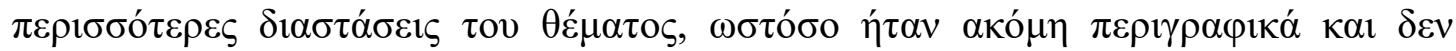

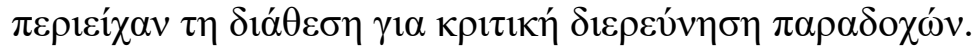

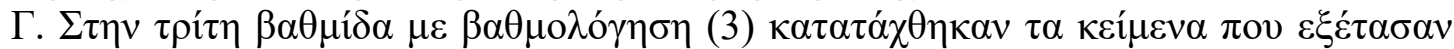

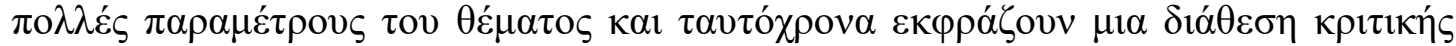

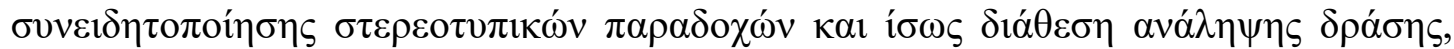

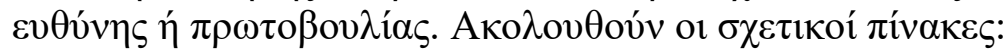

\begin{tabular}{|c|c|c|c|}
\hline $\begin{array}{l}\mathrm{A} / \mathrm{A} \\
\text { o } \mu \alpha ́ \delta \omega \nu\end{array}$ & $\begin{array}{l}\Sigma \tau \eta \nu \\
\varepsilon \dot{v} \alpha \rho \xi \eta\end{array}$ & $\begin{array}{l}\Sigma \tau \eta \\
\lambda \eta^{\prime} \xi \eta\end{array}$ & $\Delta$ ıарора́ \\
\hline $1 \eta$ & 1 & 2 & 1 \\
\hline $2 \eta$ & 1 & 1,5 & 0,5 \\
\hline 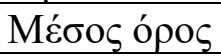 & 1 & 1,75 & 0,75 \\
\hline
\end{tabular}

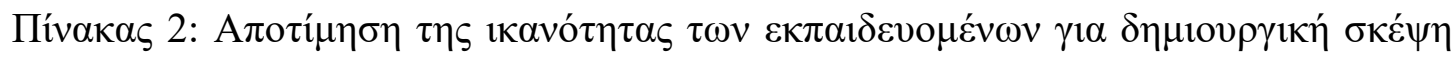

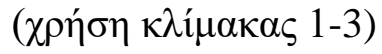

\begin{tabular}{|c|c|c|c|}
\hline 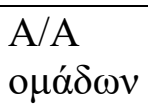 & $\begin{array}{l}\tau \eta \eta \\
\varepsilon ́ v \alpha \rho \xi \eta\end{array}$ & $\begin{array}{l}\tau \eta \eta \\
\lambda \eta \eta^{\prime} \xi \eta\end{array}$ & $\begin{array}{l}\Delta 1 \alpha \varphi \circ \rho \\
\alpha ́\end{array}$ \\
\hline $1 \eta$ & 1 & 2,5 & 1,5 \\
\hline $2 \eta$ & 1 & 1,5 & 1 \\
\hline 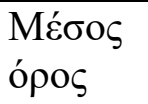 & 1 & 2 & 1,25 \\
\hline
\end{tabular}

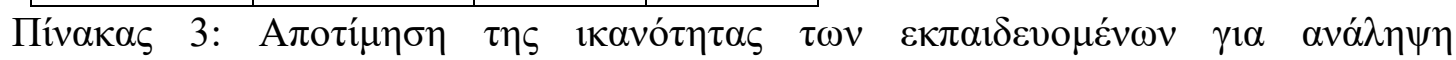

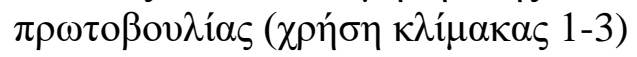

\section{4. ГYМПЕРАГМА}

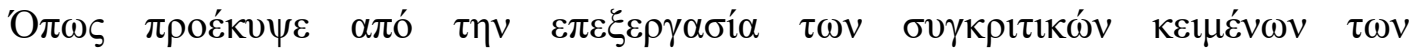

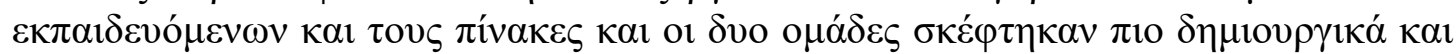




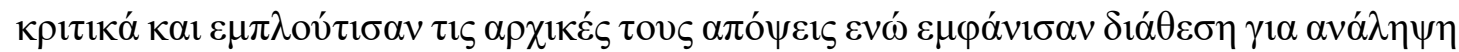

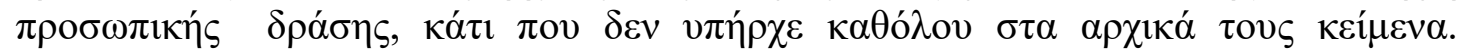

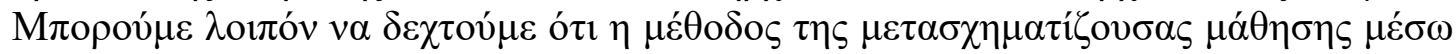

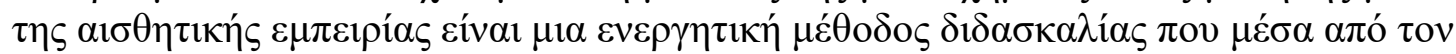

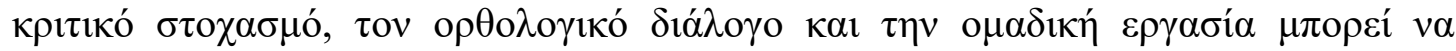

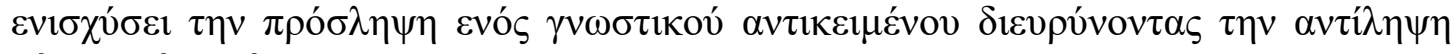

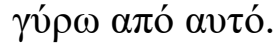




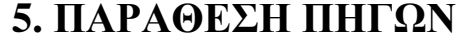

Adorno, Th. (1970).Aesthetic theory. New York: Routledge and Kegan Paul. Dewey, J. (1934 [1980]). Art as experience. USA: The Penguin Group.

Eisner, E. (2002). The arts and the creation of mind. New Haven and London: Yale UniversityPress.

Freire, P. (1970). Pedagogy of the oppressed. New York: Herder and Herder. Freire, P. (1978). Lettres á Guinée-Bissau sur l'alphabétisation. Paris: Maspero.

Gardner, H. (1983). Frames of mind: The theory of multiple intelligences. New York: Basic Books.

Gardner, H. (1990). Art education and human development. Los Angeles: The Getty Education Institute for the Arts.

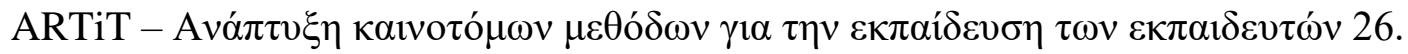

